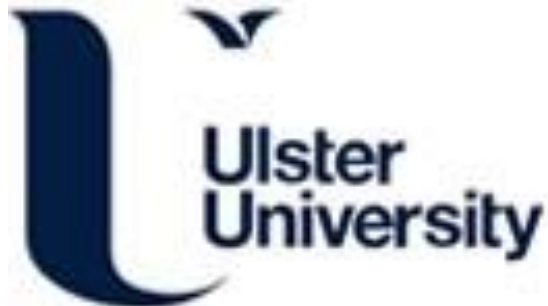

\section{Two-Level Master-Slave RFID Networks Planning via Hybrid Multiobjective Artificial Bee Colony Optimizer}

Ma, L., Wang, X., Huang, M., Lin, Z., Tian, L., \& Chen, H. (2019). Two-Level Master-Slave RFID Networks Planning via Hybrid Multiobjective Artificial Bee Colony Optimizer. IEEE Transactions on Systems, Man, and Cybernetics: Systems, 49(5), 861-880. https://doi.org/10.1109/TSMC.2017.2723483

Link to publication record in Ulster University Research Portal

Published in:

IEEE Transactions on Systems, Man, and Cybernetics: Systems

Publication Status:

Published (in print/issue): 15/04/2019

DOI:

10.1109/TSMC.2017.2723483

\section{Document Version}

Author Accepted version

\section{General rights}

Copyright for the publications made accessible via Ulster University's Research Portal is retained by the author(s) and / or other copyright owners and it is a condition of accessing these publications that users recognise and abide by the legal requirements associated with these rights.

\section{Take down policy}

The Research Portal is Ulster University's institutional repository that provides access to Ulster's research outputs. Every effort has been made to ensure that content in the Research Portal does not infringe any person's rights, or applicable UK laws. If you discover content in the Research Portal that you believe breaches copyright or violates any law, please contact pure-support@ulster.ac.uk. 


\title{
Two-level Master-slave RFID Networks Planning via Hybrid Multi-objective Artificial Bee Colony Optimizer
}

\author{
Lianbo Ma ${ }^{\mathrm{a}}$, Xingwei Wang ${ }^{\mathrm{a}}$, Min Huang ${ }^{\mathrm{b}}$, Zhiwei Lin ${ }^{\mathrm{c}}$, Liwei Tian ${ }^{\mathrm{d}}$, Hanning Chen ${ }^{\mathrm{e}}$ \\ ${ }^{a}$ College of Software, Northeastern University, Shenyang 110819, China \\ ${ }^{\mathrm{b}}$ College of Information Science and Engineering, Northeastern University, Shenyang 110819, China \\ ${ }^{\mathrm{c}}$ School of Computing and Mathematics, Ulster University, BT37 0QB UK \\ ${ }^{\mathrm{d}}$ Shenyang University, Shenyang, 110044, China \\ ${ }^{\mathrm{e}}$ School of Computer Science and Software, Tianjin Polytechnic University, 300387, Tianjin, China
}

\begin{abstract}
RFID networks planning (RNP) is a challenging task on how to deploy RFID readers under certain constraints. Existing RNP models are usually derived from the flat and centralized-processing framework identified by vertical integration within a set of objectives which couple different types of control variables. This paper proposes a two-level RNP model based on the hierarchical decoupling principle to reduce computational complexity, in which the cost-efficient planning at the top levels is modeled with a set of discrete control variables (i.e., switch states of readers), and the QoS (quality of service) objectives at the bottom level are modeled with a set of continuous control variables (i.e., physical coordinate and radiate power). The model of the objectives at the two levels is essentially a multi-objective problem. In order to optimize this model, this paper proposes a specific multi-objective artificial bee colony optimizer called $\mathrm{H}-\mathrm{MOABC}$, which is based on performance indicators with reinforcement learning and orthogonal Latin squares approach. The proposed algorithm proves to be competitive in dealing with two-objective and three-objective optimization problems in comparison with state-of-the-art algorithms. In the experiments, H-MOABC is employed to solve the two scalable real-world RNP instances in the hierarchical decoupling manner. Computational results shows that the proposed $\mathrm{H}-\mathrm{MOABC}$ is very effective and efficient in RFID networks optimization.
\end{abstract}

Index Terms-Multi-objective Algorithm, Multi-level RNP, H-MOABC.

\section{INTRODUCTION}

In recent years, radio frequency identification (RFID) networks have become increasingly prevalent in the mainstream applications of Internet of Things (IoT), including retail production monitoring, supply chain management, localization and navigation [1-4]. It is desirable to detect and recognize all tags located in the workspace by using the RFID readers and their corresponding parameters, which are optimized. The task on how to deploy RFID readers and optimize the parameters is the well-known RFID networks planning (RNP) problem [3-8]. However, the RNP is very challenging due to the complexity of the conditions imposed by the large-scale RFID networks. First, the emerging large-scale RFID systems involve a rapidly increasing number of readers and tags, which have to be systematically deployed in an efficient manner. Therefore, given a specific RNP, the dimension and its mutual coupling degrees of the RNP problem could be very large. In other words, the complexity increases in proportion to the scale of the RFID network. Second, there are different types of underlying optimization goals or decision variables to couple together, including discrete and continuous variables (e.g., complete coverage, high QoS and cost efficiency). The complexity for optimization increases dramatically when the discrete decision variables and continuous variables need to be mutually coupled. The heterogeneous nature of the variables with continuous and discrete control variables in RNP poses great challenge for optimization. For example, the control variables (e.g., the state of a single device) and the minimal number of readers to be deployed in RNP are discrete while the limited detection range of single device, $x-y$ coordinates of the devices in the network are continuous. Optimizing an RNP model with a mixture of heterogeneous variables of the number of multiple objectives, mixed control variables and constrains is not trivial and has been proven to be an NPhard issue $[6,7]$.

The RNP problem is a specific instance of sensor deployment problem in wireless sensor networks (WSN for short), when the electronic tags and readers in RFID network are treated as a special class of wireless sensors. However, the RNP problem, strictly speaking, is different from other WSN senor deployment problems (e.g., the mobile phone network, Ad Hoc network) in aspects of controlled object, controlled variable and control type. First, the main difference lies in the fact that a WSN is a multi-hop node-to-node communication model, while in an RFID network, the RFID tags (nodes) are only detected passively by the readers within a limited interrogation range $[7,8]$. This implies that the controlled object in the RNP is the optimal parameter configure of deployed readers (yet not the tags) involving both continuous and discrete variables to potentially enhance the reader's performance in the given region, unlike that the WSN needs to optimize the parameters of every accessible nodes. Second, the controlled variables in the RNP usually involve the static variables, such as the amount of readers, the locations and radiated power of readers [9]. Due to its random mobility and dynamic topology, the WSN deployment usually involves the time-varying variables. Third, compared with other WSN scenarios, in RFID system, the interference between readers and tags is more complicated, and the installment cost relies more heavily on the number of deployed devices. Consequently, the optimal cost efficiency without any communication interference is more essential and urgent in the RNP problem.

Several models have been proposed for the RNP problems. The state-of-theart methods formulate the RNP problem as a set of singe-objective with continuous variables $[8-12,18-24]$. These objectives are combined into a compositional function by the weighted-sum approach so that they can be optimized by the conventional intelligent algorithms. Such methods need extrawork to determine specific coefficients for the compositional function, and they cannot handle the trade-off between some conflicting objectives. So far, there are only a very limited number of studies $[16,17]$ to apply multi-objective Pareto-based scheme to solve RNP, whereas they only can handle RNP problems with continuous variables but they have not considered priority and dependency between tasks. Note that the aforementioned RNP schemes are all based on the one-level or flat optimization framework, also called vertical integration, which couples different RNP objectives together in an optimization container. One drawback is that such framework cannot deal with complex RNP scenarios with high coupling degree where the RNP objectives have different types of decision variables, different dimensions and different corresponding priorities. Also, with the increasing of the number of the involved objectives, the complexity of coupling action between different objectives grows exponentially. Therefore, the existing RNP frameworks with coupling actions are ineffective in the complex RFID scenarios.

In this paper, based on the principle of distributed decision making (DDM) [24], we propose a multi-level RNP optimization framework to decouple complex RNP problems, which has significant features of centralized control, flexibility and simplification. Different from the previous works [13-23], in this hierarchical framework, various objectives are assigned to the corresponding target level and can be optimized by specific effective strategies according to the decision variable's type and task priorities. In our framework, to eliminate redundant readers, a two-level multi-objective RNP (MORNP) model is instantiated, in which the binary optimizer at the top level aims to minimize the number of readers with maximal coverage, and the bi-objective and triobjective optimization schemes at the bottom-level are respectively optimized in order to provide optimal position and radiated power for each readers.

To achieve the above goals with the MORNP model, this paper exploits a specific RNP-oriented multi-objective evolutionary optimizer named as $\mathrm{H}$ MOABC to optimize the proposed multi-level RNP model effectively, which combines the foraging rules of artificial bee colony ( $\mathrm{ABC}$ ) paradigm with the quality indicator based fitness evaluation and specific multi-objective techniques. Within $\mathrm{H}-\mathrm{MOABC}$, an external archive based on both Pareto dominance and preference indicators is designed to preserve the non-dominated solutions in each generation, and then a novel dynamical indicator-based crowded comparison (DICC) mechanism is developed to maintain this archive. By incorporating these mechanisms, the $\mathrm{H}-\mathrm{MOABC}$ can search a set of representative Pareto optimal solutions with a remarkable approximation as well as a uniform spread.

The contribution of this paper lies in:

(1) A novel two-level master-slave RNP framework and instantiated model are proposed to break down conventional vertical integration by using the hierarchical decoupling approach.

- A complicated RNP problems can be decomposed into two types of instances: the continuous RNP instances and the discrete RNP instances, where the continuous RNP instances will be solved at the 
bottom layer and the discrete instances will be solved at the top layer so that they will be cooperatively optimized in a decoupling manner. These RNP instances at different levels are optimized in a certain order according to their priorities. For example, the master optimizer at the top level generally is designed for the scheduling task with higher priorities, and the slave optimizer at the bottom layer is implemented for the basic RNP operations. The proposed framework is very flexible to allow further integration of new RNP objectives and new evolutionary algorithm (EA) or swarm intelligence (SI) approaches.

- Based on our proposed framework, a two-level MORNP model is instantiated to decouple conventional RNP optimization into binary top-level scheduling and continuous bottom-level planning. Specifically, the top-level scheme is implemented to eliminate redundant readers for reducing cost. And the bottom-level scheme is responsible for optimizing different continuous conflicting objectives simultaneously, including tag coverage, economic efficiency, load balance, and reader interference. By contrast to conventional RNP models, this hierarchical processing can dynamically eliminate redundant readers during the optimization process, and also reduces the optimization complexity.

(2) In order to solve our proposed RNP model effectively, a novel RNPorient $\mathrm{ABC}$-based optimizer called $\mathrm{H}-\mathrm{MOABC}$ is developed by using quality indicator based multi-objective strategies. This algorithm is added into the algorithm library of this RNP framework

- The preference information based on a quality indicator $I$ is used. The new fitness assignment based on quality indicator is designed to update the individuals in the population. In this approach, the population members are evaluated and ranked according to their usefulness in relation to the optimization goal.

- We propose to maintain the external archive by synthetically incorporating the spatial Euclidean distance and the logical performance improvement indicator. Within the DICC, only two solutions are involved to perform re-calculation of crowding distance in each deleting operation, which significantly reduces the computation complexity.

- The reinforcement learning (RL) based on Q-function is incorporated into the single-objective $\mathrm{ABC}$ paradigm to determine the flight trajectories of the foraging bee in order to improve the efficiency of information exchange. Also, the orthogonal Latin squares and comprehensive learning are used in the population initiation and searching process to avoid being trapped into local Pareto fronts.

The rest of this paper is organized as follows. Section 2 overviews the related works. In Section 3, a multi-level RNP framework is proposed and two-level MORNP model is instantiated. Section 4 presents the H-MOABC algorithm in detail and the corresponding experimental studies. In Section 5 , the implementation of H-MOABC on two RNP instances- $C d 100$ and $R d 500$ is detailed, and the experimental results are analyzed. Finally, in Section 6 conclusions are outlined.

\section{RELATED WORKS}

The primary requirement for a well-planned RFID system is that each tag should be detected and recognized by at least one reader or sensor without reader-to-reader collision [12]. Most of recent work mainly focuses on some of the RNP objectives such as tag coverage, interference, load balance and etc [820]. For example, the objective for coverage is to identify all target tags within a limited working region by arranging minimal number of readers. The objective for interference aims to reduce the chance of collision between readers in a densely region. However, when combining several objectives in a single RNP, it is difficult to obtain an optimal solution as the optimization complexity for multi-objective is very high.

From the information management point of view, existing RNP models are usually based on the coupling vertical integration framework by taking into account continuous variables only [8-12]. For examples, the very early work in single-objective RNP model is studied in [8], and this static RNP model is further formulated as a non-linear optimization problem in [9-11]. Kumar et al. develops an RFID embedded model to map the economical merits in closed loop logistics [12,13], and then examines the economical impact of RFID adoption to remanufacturing [14]. Especially, a k-coverage RNP model is formulated as a multi-dimensional optimization problem with constraint conditions, to evaluate the network performance. Furthermore, a dynamic RNP model with elimination of redundant readers is developed by Y. Gong et al. and this model is handled with by an improved PSO method with tentative reader elimination (TRE), which has gained significant attentions [15]. These models usually transform the involved RNP objectives into one combinatorial function through the weight-sum method and then solve it by a single-objective algorithm. Some recent studies $[16,17]$ have developed multi-objective RNP optimization schemes based on Pareto optimality however they require the control variables to be continuous variables without any priority. All these RNP schemes are based on the flat-coupling optimization framework and they ignore complex coupling actions of various objectives with different priorities and variable types. As a result, this flat-coupling scheme is very ineffective with the increasing number of objectives and of the types of decision variables. For example, in a large-scale RFID system for supply chain management, the installation cost reduction relies largely on minimizing the number of the placed RFID devices and this objective is of high priority. Obviously, this objective involves a discrete variable, which is very incompatible with the other RNP goals with continuous variables, e.g., coverage. As such, based on the fact that an RNP has hybrid variables, an RNP is a complicated multi-objective (MO) and mixed-continuous-discrete optimization problem.

The EA and SI algorithms have been widely applied to resolve these RNP models due to their flexibility and robustness. In [18, 19], the differential evolution (DE) algorithm and particle swarm optimization (PSO) algorithm are firstly introduced to the RNP optimization [18], and the genetic algorithm (GA) is also directly applied for the RFID network planning [20]. The bacteria foraging optimization (BFO) algorithm has also been used to find the optimal layout and deployment for RFID system [21]. The study in [22] proposed a new anti-collision algorithm based on artificial immune network for optimizing the deployment of readers. In [10], a novel bio-inspired algorithm named as plant growth simulation algorithm is proposed to solve the k-coverage RNP model. The application of $\mathrm{ABC}$ algorithm and its variants to optimize the optimal locations of dense sensors has achieved satisfying results [23-24]. The ABC algorithm is an effective evolutionary computation paradigm inspired from the intelligent foraging behavior of honeybees and has exhibited enormous potential in a variety of complex optimization applications [25-28]. Recently, several works have extended the $\mathrm{ABC}$ algorithm to tackle multi-objective problems. In [25], three multi-objective $A B C$ variants using synchronous and asynchronous models, namely A-MOABC/PD, A-MOABC/NS and S$\mathrm{MOABC} / \mathrm{NS}$, are proposed by incorporating Pareto-dominance and nondominated sorting, and experimentally validated on a set of unconstrained CEC09 benchmarks. In [26], a generic model based on the vector-evaluated $\mathrm{ABC}$ (VEABC) algorithm is developed to resolve the design optimization of laminated composite components. In [27], a dynamic multi-colony multiobjective $\mathrm{ABC}$ called DMCMOABC is designed by using the multi-deme evolution and dynamic information exchanging strategies. In [28], a new multiobjective $\mathrm{ABC}$ variant based on external archive is developed by introducing an elitism strategy, which is conductive to avoid premature convergence. In [29], the elite-guided multi-objective $\mathrm{ABC}$ algorithm is proposed by employing the elite-guided solution generation strategy to exploit the neighborhood of existing solutions based on the guidance of the elite.

The above MOABCs are based on Pareto dominance, which is widely used to determine the non-dominated solutions in the obtained population [58]. However, these approaches are not flexible for the use of preference information [37]. It has been shown that, in some specific cases, these Paretobased MO algorithms suffer from poor convergence caused by the loss of selection pressure [59]. Unlike the abovementioned Pareto-based MOABCs, in our $\mathrm{H}-\mathrm{MOABC}$, we propose to use the preference information indicator (i.e., the binary quality indicator $I$ in [37]) to evaluate the dominance relations of obtained solutions, which essentially represents a different type of non-Paretobased approaches. Accordingly, the main difference between H-MOABC and existing MOABCs lies in the fact that $\mathrm{H}-\mathrm{MOABC}$ stresses the importance of the contribution or usefulness of individual in the population to the optimization goal, which is ranked based on the indicator-based fitness assignment with good convergence performance, further develops the DICC mechanism for diversity presentation, and then utilizes RL strategy to enhance the individual's information exchange.

\section{MUlti-level RNP FramewORK AND MOdel}

\subsection{RFID Deployment Modeling}

In a traditional RFID system, the tags and readers are the two main components to be deployed optimally in a specific work region. Specifically, a tag is attached to a physical object to be tracked with a unique ID for the associated object. Wireless communication is established between the readers and the tags with a limited interrogation range. The tags, based on EPC protocol, are in passive model. The proposed RNP model aims to improve the QoS of the RFID network (e.g., tag coverage, reader interface, economic efficiency and load balance) by tuning the system's parameters, including the number, location, and radiated power of readers. With regard to the frequency of operations for RFID network, the reader-to-tag communicating or recognizing is a highfrequency operation, which is activated immediately once the reader sends reading/wring command to its ambient tags. In addition, it is also necessary to re-optimize the deployment of the readers and the corresponding parameters if the system detects that new tags join or the existing tags leave the working region.

To establish a robust communication between a reader and a tag, both the reader-to-tag communication and tag-to-reader communication must be taken into account together [12]. For tag-to-reader communication, the radio signal propagation between readers and tags via RF antennas would cause 
transmission link budget. As studied in [12], through the RF communication process, the final power $P_{t}$ received by one targeted tag from a reader can be calculated as:

$$
\begin{aligned}
& P_{t}=P_{\mathrm{o} 1}+G_{r}+G_{t}-L, \\
& L=10 \log \left[\left(\frac{4 \pi}{\lambda}\right)^{2} d^{n}\right]+\delta
\end{aligned}
$$

where $P_{o l}(\mathrm{dBm})$ is the output transmitted power of the reader, $G_{r}(\mathrm{dBi})$ and $G_{l}$ $(\mathrm{dBi})$ are the antenna gain of the reader and the targeted tag, respectively, and $L(\mathrm{~dB})$ is the attenuation factor. The variable $L$ is calculated by Friis transmission equation $[8,12,30]$, where $\lambda$ is the transmitted wavelength, $d^{n}$ is the distance between the two devices, $n$ is a adjustable coefficient, and $\delta$ represents other influence factors.

Similarly, based on Friis equation $[12,30]$, the final power received by one reader from an activated tag is calculated as:

$$
P_{r}=P_{\mathrm{o} 2}+G_{t}+G_{r}-20 \log \left(\frac{4 \pi}{\lambda}\right)^{2}, P_{02}=\left(\tau_{\text {tag }}\right)^{2} P_{o 1}
$$

where $G_{r}$ and $G_{t}(\mathrm{dBi})$ are the antenna gain of the reader and the tag, respectively, $P_{\mathrm{O} 2}(\mathrm{dBm})$ is the backscatter power transmitted by the tag, and $\tau_{\text {tag }}$ is the reflection coefficient of the tag. Before constructing RNP objective functions, the following notations and presentation used in RNP are

\begin{tabular}{|c|c|c|c|}
\hline$R S:$ & $\begin{array}{l}\text { the set of readers deployed in } \\
\text { the workspace }\end{array}$ & $T S:$ & $\begin{array}{l}\text { the set of tags located in the } \\
\text { workspace }\end{array}$ \\
\hline$P_{r, t}:$ & $\begin{array}{l}\text { the radio signal power } \\
\text { received by tag } t \text { from reader } r\end{array}$ & $P_{t, r}:$ & $\begin{array}{l}\text { the radio signal power received } \\
\text { by reader } r \text { from tag } t \text {. }\end{array}$ \\
\hline$P_{T}:$ & $\begin{array}{l}\text { the threshold value to reader- } \\
\text { to-tag communication }\end{array}$ & $P_{R}$ & $\begin{array}{l}\text { the threshold value to build } \\
\text { reader-to-tag communication }\end{array}$ \\
\hline$N_{r}:$ & $\begin{array}{l}\text { the number of the readers } \\
\text { deployed in the workspace }\end{array}$ & $N_{t}$ & $\begin{array}{l}\text { the number of the tags located in } \\
\text { the workspace }\end{array}$ \\
\hline$N_{\max }:$ & $\begin{array}{l}\text { the maximum number of the } \\
\text { readers that can be deployed }\end{array}$ & & \\
\hline
\end{tabular}
summarized in Table 1.

Table 1. Notations for RNP model

Tag coverage $\left(\boldsymbol{f}_{1}\right)$ : This is an objective for the coverage of all the tags and we assume that all the tags in the working region should be covered at least by one reader. In order to achieve this objective, for any tag $t$ from $T S$, the received power $P_{r l, t}$ of one tag $t$ from reader $r_{I}\left(r_{1} \in R S\right)$ should exceed the threshold $P_{T}$ to establish the reader-to-tag communication while the backscatter signal $P_{t, r l}$ received by reader $r_{l}$ should exceed the threshold $P_{R}$ to ensure the available tag-to-reader communication. Here the $P_{r l, t}$ and $P_{t, r l}$ can be calculated by Eq. (1) and Eq. (2) respectively. Then, the objective function of tag coverage can be formulated as

$$
\begin{aligned}
& f_{1}=\max \sum_{t \in T S} \frac{\operatorname{Cov}(t)}{N_{t}} \\
& \operatorname{Cov}(t)=\left\{\begin{array}{l}
1, \text { if } \exists r 1 \in R S, t \in T S, P_{r 1, t}>P_{T} \wedge P_{t, r 1}>P_{R} \\
0, \text { otherelse }
\end{array}\right.
\end{aligned}
$$

Reader interference $\left(f_{2}\right)$ : There are inevitably reader collision accidents once multiple densely deployed readers try to interrogate a tag at the same time. This interference will deteriorate system performance. Therefore, minimizing the chance of collision between the readers is an important objective in the RNP. The total amount of interference in an RFID network is formulated as the sum of the interference value at each tag, as follows:

$$
f_{2}=\min \sum_{t \in T S} \sum_{r \in T S}\left(P_{r, t}-\max \left(P_{r, t}\right)\right), P_{r, t} \geq P_{T}
$$

Economic Efficiency $\left(f_{3}\right)$ : Consider multi-path propagation loss, channe attenuation and the stochastic noise, readers should be deployed near the center of a tag cluster. Thus, this objective function is calculated by weighing the distances of each center of tag clusters from its best served reader, and it can be formulated as

$$
f_{3}=\min \sum_{r \in R S} \operatorname{dist}\left(I_{r}, \theta_{r}\right)
$$

where $\operatorname{dist}()$ is the distance between reader $r$ and the corresponding tag center, $\theta_{r}$ is the position of cluster center of reader $r$ and $I_{r}$ is the best served reader.

Load Balance $\left(\boldsymbol{f}_{4}\right)$ : In order to boost the efficiency of energy saving, it is significant to devise an effective load balancing scheme to distribute tags among readers as uniformly as possible. That is, a network with a welldistribution of readers can provide a better performance than an a network with poor configuration. In a large-scale RFID deployment scenario, the set of distributed tags to be sensed should be appropriately balanced among all deployed readers. This objective function for minimizing the variance of load conditions is defined as

$$
f_{4}=\min \prod_{r \in R S}\left(\frac{C_{r}^{\max }}{C_{r}}\right)
$$

where $C_{r}$ is the number of tags assigned to reader $r$ and $C_{r}^{\max }$ is the maximum number of tags interrogated by the reader $r$ in an unit time.
Objective Constraint: All tags located in working region should be covered by one reader. This constraint is formulated as:

$$
\text { s.t. } \mathrm{P}_{\mathrm{r}, t} \geq P_{T}, P_{t, r} \geq P_{R} \quad \forall t \in T S, r \in R S
$$

$$
\sum_{r \in R S} C_{r}^{t} \geq 1 \quad \forall t \in T S
$$

where $C_{r}^{\mathrm{t}}$ is the number of available readers that ensure a complete coverage. Note that $C_{r}^{\mathrm{t}}=1$ if the reader $\mathrm{t} \in R S_{i}$, otherwise $C_{r}^{\mathrm{t}}=0$.

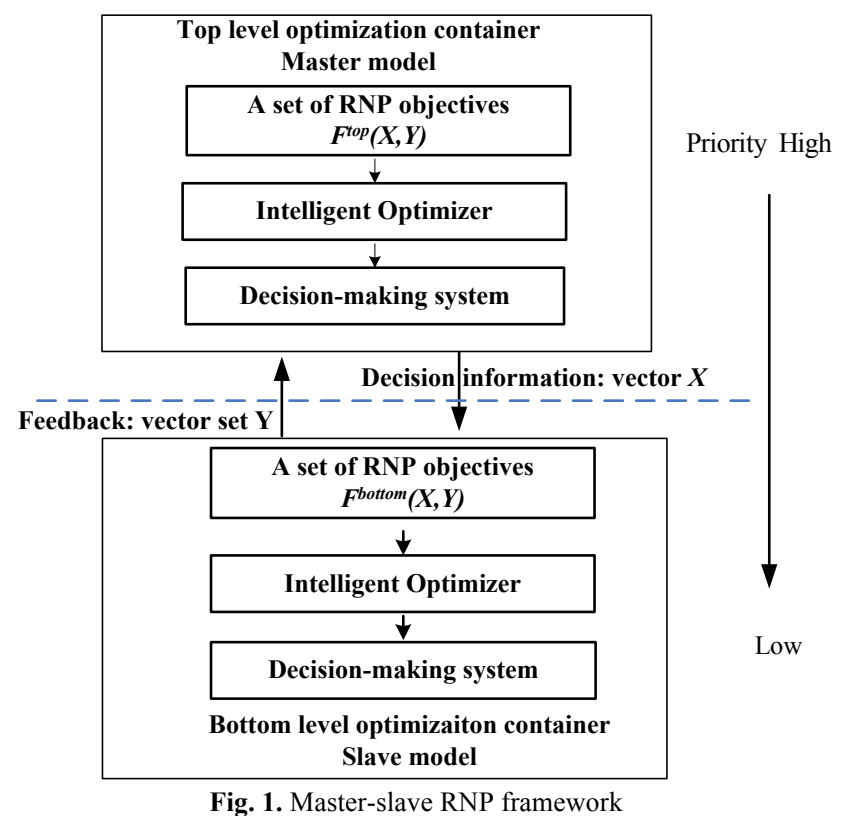

\subsection{Two-level Master-slave RNP Framework and Corresponding Model}

In this section, based on the distributed decision making (DDM) theory [31, 32], a two-level master-slave RNP framework is developed as shown in Fig.1. In this framework, multiple RNP objectives are grouped into the two layers according to implementation priority or variable's type of objectives, and optimized by the specific optimization scheme, respectively. Generally, the master model in the top layer has higher priority and provides a decision information to guide the optimization process of the slave model in the bottom layer. And then, the slave model feeds back to its upper layer.

From Fig.1, the general mathematical formulation of the RNP objectives can be defined as

$$
\begin{aligned}
& \left(\min _{x}\left(F_{1}^{t o p}\left(x, y_{1}, y_{2}, \cdots, y_{N}\right), \cdots, F_{m}^{t o p}\left(x, y_{1}, y_{2}, \cdots, y_{N}\right)\right)\right. \\
& \text { s.t. } \\
& G\left(x, y_{1}, y_{2}, \cdots, y_{N}\right) \leq 0 \\
& H\left(x, y_{1}, y_{2}, \cdots, y_{N}\right)=0 \\
& \left\{\begin{aligned}
\min _{y_{i}} & \left(F^{\text {bottom }}\left(x, y_{i}\right), F^{\text {bottom }}\left(x, y_{i}\right), \cdots, F_{k}^{\text {bottom }}\left(x, y_{i}\right)\right) \\
& \quad g\left(x, y_{1}, y_{2}, \cdots, y_{N}\right) \leq 0 \\
& h\left(x, y_{1}, y_{2}, \cdots, y_{N}\right)=0
\end{aligned}\right.
\end{aligned}
$$

where $m$ is the number of objectives in top layer and $k$ is the number of objectives in bottom layer, and

$$
\begin{aligned}
& x=\left(x_{1}, \ldots, x_{n_{0}}\right) \in R^{n_{0}}, y_{i} \in R^{n_{i}}, i=1,2, \ldots, N \\
& F\left(\mathrm{x}, \mathrm{y}_{1}, \ldots, \mathrm{y}_{\mathrm{N}}\right): R^{n_{0}} \times R^{n_{1}} \times \ldots \times R^{n_{N}} \rightarrow R \\
& G=\left(G_{1}, \ldots, G_{P}\right)^{T}: R^{n_{0}} \times R^{n_{1}} \times \ldots \times R^{n_{N}} \rightarrow R^{P} \\
& H=\left(H_{1}, \ldots, H_{Q}\right)^{T}: R^{n_{0}} \times R^{n_{1}} \times \ldots \times R^{n_{N}} \rightarrow R^{Q} \\
& f_{i}\left(x, y_{1}, \ldots, y_{N}\right): R^{n_{0}} \times R^{n_{1}} \times \ldots \times R^{n_{N}} \rightarrow R, i=1,2, \ldots, N \\
& g_{i}\left(g_{i 1}, \ldots, g_{i p_{i}}\right)^{\mathrm{T}}: R^{n_{0}} \times R^{n_{1}} \times \ldots \times R^{n_{N}} \rightarrow R^{p i}, i=1,2, \ldots, N \\
& h_{i}\left(h_{i 1}, \ldots, h_{i q_{i}}\right)^{\mathrm{T}}: R^{n_{0}} \times R^{n_{1}} \times \ldots \times R^{n_{N}} \rightarrow R^{q i}, i=1,2, \ldots, N
\end{aligned}
$$

In the iterative optimization process, the master optimizer firstly provides an initial decision vector $x$ to satisfy the constrict of Eq.(8). This vector will be taken as a prerequisite to guide the optimization of the slave model. Then, under the premise of $x$, the slave optimizer searches the optimal vector $y$ to minimize the objective with its constrict. By using the feedback vector $y$, the master optimizer will further adjust its decision $x$ till its objectives with the constrict reach the optimal state.

Because the RFID installation cost relies heavily on the number of readers to be deployed, minimizing the deployed reader number has become a challenging task with high priority. Thus, an actual two-level RNP model is instantiated as 
shown in Fig. 2. This model includes top-level scheduling for discrete control variables and bottom-level planning for continuous control variables. In this framework, the top-level optimizer is responsible to minimize the number of deployed readers by dynamically eliminating redundant readers in a binary manner, and the bottom-level optimizes continuous objective functions simultaneously in the multi-objective manner.

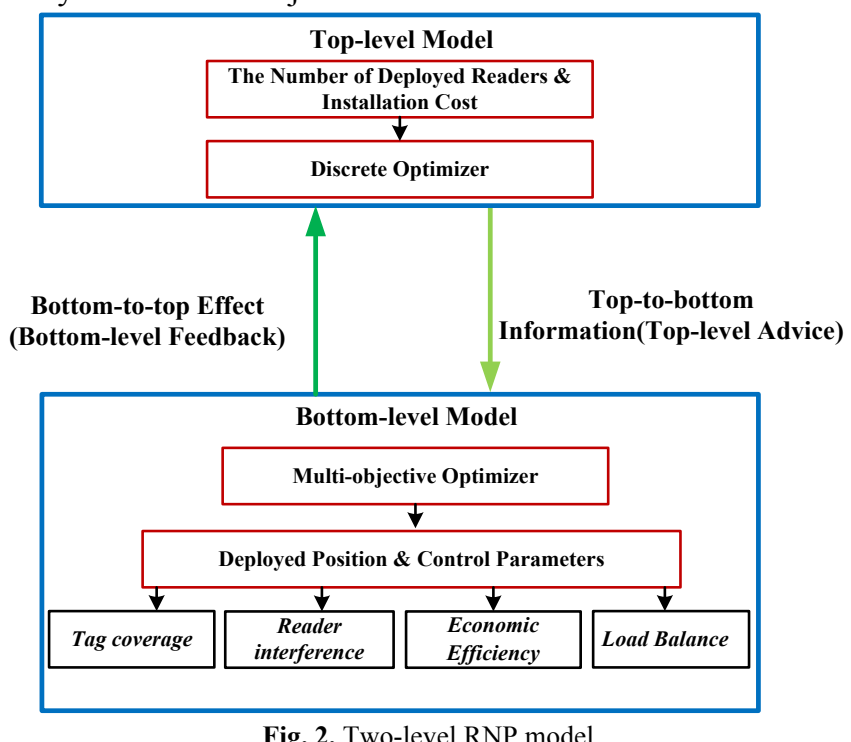

Fig. 2. Two-level RNP model

At the top-level, decision variables are defined as a discrete vector $R^{T}=\left(r_{1}, r_{2}, \ldots, \mathrm{r}_{\text {Nmax }}\right)$ to represent the array of deployed readers and each element $r_{i} \in[0,1], i=1,2, \ldots, N_{\max }$. Here, " 0 " indicates the corresponding reader is redundant, and " 1 " means the corresponding reader is available. Then the toplevel model can be defined as below:

$$
F_{\text {Top }}\left(R^{T}\right)=\min _{R} \sum_{i=1}^{M} w_{i} f_{i}\left(R^{T}\right) \text {, s.t. } \sum_{j=1}^{N_{\max }} c_{j} r_{j} \leq I_{\max }
$$

where $f_{j}$ is the $j$-th RNP objective function, $M$ is the number of objective functions, $w_{i}$ is the weighting factor of $i$-th objective function, $c_{i}$ donates the cost of reader $j$, and $I_{\max }$ is the maximum total cost.

According to the optimal reader number from the top-level, the bottom-level multi-objective optimizer handles different systematical objectives simultaneously via regulating continuous variables of each reader. Then, the bottom-level decision variables $X$ can be transformed as shown in Table 2 and the corresponding multi-objective problems can be defined as below:

$$
F_{\text {Bottom }}(X)=\min _{X}\left\{f_{1}(X), f_{2}(X) \ldots, f_{M}(X)\right\}
$$

\section{- Solution presentation}

In the top-level optimization, the solution of the readers can be encoded as a binary vector, as shown in Table 2 . The corresponding bit state of the binary vector represents the presence or absence of the $i$-th reader. That is, a bit ' 0 ' in a solution vector donates the corresponding reader is redundant, while a bit ' 1 ' indicates the essential reader should be deployed. During the top-level optimization, each bit state of a solution vector evolves with the time steps to enable the reader number to be changed dynamically, and then the redundant readers can be removed.

In the bottom-level optimization, two spatial variables and one functional variable of readers are about to be adjusted in the RFID system as shown in Fig.3:

$\mathbf{X}$ : the $\mathrm{x}$ axis coordinate of one deployed reader.

$\mathbf{Y}$ : the $y$ axis coordinate of one deployed reader.

P: the interrogation range (i.e. radiate power level) of one deployed reader.

Then, in the RNP optimization, these RFID variables can be encoded into a unified representation of the optimization solution as shown in Table 3. That is, each solution can be represented by a $D$-dimensional vector where $D=3 N_{r}$. In this representation, the first $N_{r}$ dimensions represent the x coordinates of deployed readers, the second $N_{r}$ dimensions are the y coordinates of deployed readers, and the rest $N_{r}$ dimensions donate the radiate power level of each reader. Table 2. Unified representation of top-level solution.

\begin{tabular}{ccccccccccc}
\hline & \multicolumn{1}{c}{ Pre-deployed readers } \\
\hline Solution vector $i$ & $X_{i}^{l}$ & \multicolumn{1}{c}{$X_{i}^{2}$} & $X_{i}^{3}$ & $\ldots$ & $X_{i}^{N r}$ \\
Value & 0 & 1 & 0 & 1 & 0 & 1 & & 0 & 1 \\
\hline
\end{tabular}

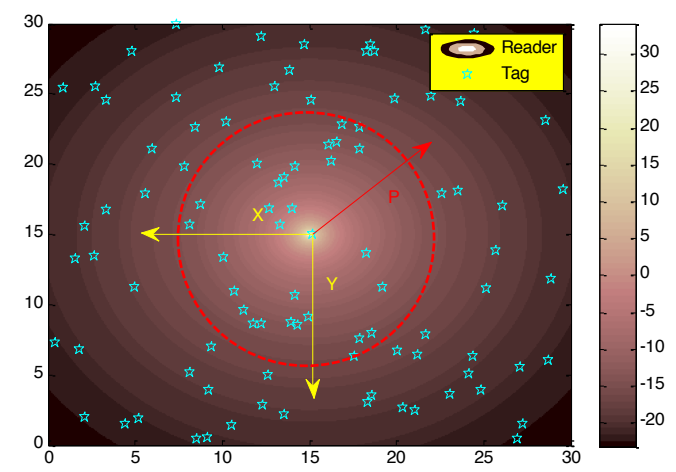

Fig. 3. A typical RFID reader deployment scenario Table 3. Unified representation of bottom-level solution.

\begin{tabular}{lllllllllll} 
& \multicolumn{3}{c}{ X-coordinate } & \multicolumn{3}{c}{ Y-coordinate } & \multicolumn{2}{c}{ P-coordinate } \\
\hline Solution vector $i$ & $X_{i}^{l}$ & $\ldots$ & $X_{i}^{N r}$ & $Y_{I}^{r}$ & $\ldots$ & $Y_{i}^{N r}$ & $P_{i}^{l}$ & $\ldots$ & $P_{i}^{N r}$
\end{tabular}

\section{Hybrid MUlti-OBJeCtive ARTIFiCIAL BEe Colony Optimizer}

The original ABC algorithm initially proposed in [33] has been deeply developed via learning from the intelligent cooperative foraging mechanism of three types of honeybees, i.e., employed bee, onlooker bee and scout bee. Recent studies [28-33] have validated the great potential of ABC to solve conventional optimization problems, such as sub-optima trap, slow convergence and dimension coupling. To take advantage of the $\mathrm{ABC}$, the proposed $\mathrm{H}-\mathrm{MOABC}$ algorithm deliberately restructures the foraging rules of bees with comprehensive learning and orthogonal Latin squares approach to strengthen the efficiency of population information exchanging. Moreover, unlike abovementioned MOABCs based on Pareto dominance, the H-MOABC incorporates binary quality indicator to evaluate the quality of candidate solutions and then utilizes an external archive to memorize non-dominated solutions previously found. This proposed fitness assignment using binary quality indicator as dominance preserving can amplify the influence of dominating population members over the dominated ones. Given the fixed archive size, a novel dynamical indicator-based crowded comparison (DICC) mechanism based on indicator-based calculation and Pareto dominance is designed to keep diversity preservation. The main components of the algorithm can be given as below.

\subsection{Algorithm}

\subsubsection{Neighbor-discount-information mechanism}

In RL, the Q-learning is used step-by-step to determine an optimal strategy which maximizes the total discounted expected reward in future [34]. Let $S=\left[\mathrm{s}_{1}\right.$, $\left.s_{2}, \ldots, s_{n}\right]$ be a series of available states for each agent, $A=\left[a_{1}, a_{2}, \ldots, a_{n}\right]$ be a set of available actions to be executed, $r_{t}$ be the immediate reward for implementing actions $a_{t}$, the transition rule of Q-learning can be defined as

$$
Q(s, a)=r_{t}+\gamma \max _{a}\{Q(s, a)\}
$$

Each learning agent selects an action by observing the state vector and then enters next state, then in order to obtain the maximum return rewards at next state, the main procedures of Q-learning follow as: (1) observe current state $s_{t}$, (2) select and implement an action $a_{t}$, (3) receive a immediate reward $r_{t}$, and (4) adjust the $\mathrm{Q}$ value as

$$
Q_{t}\left(s_{t}, a_{t}\right)=\left(1-\alpha_{t}\right) Q_{t-1}\left(s_{t}, a_{t}\right)+\alpha_{t}\left[r_{t}+\gamma \max _{a \in A} Q\left(s_{t+1}, a\right)\right]
$$

where $a_{t}$ is the learning rate to control the learning speed, the discount factor $\gamma$ is set within $[0,1]$ to ensure the convergence of the $\mathrm{Q}$ function. It has been proven that the Q-learning will converge if the condition of $a$ is met in a Markov environment [34].

ID: Communicate Directly

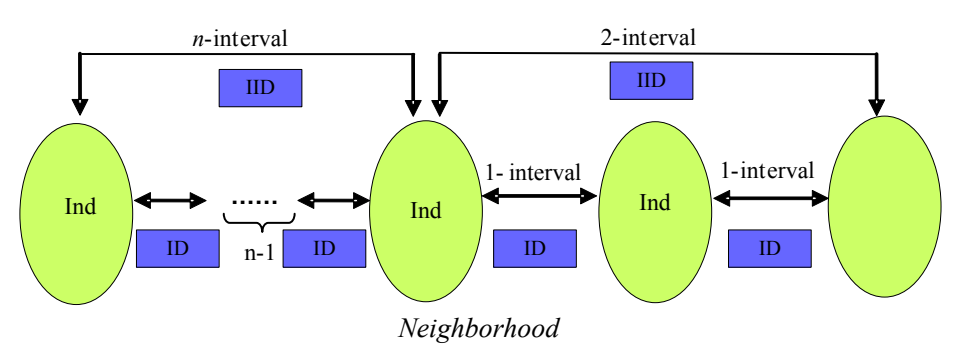

Fig. 4. Interaction relationships of individuals in neighborhood 
In order to enhance the efficiency of information exchange in the population, the neighbor-discount-information (NDI) learning mechanism is developed based on RL.

Definition 1. Given an individual Ind, the individual that can communicate

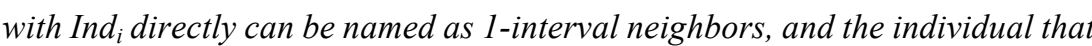
can only communicate with 1-interval neighbors directly can be called 2interval neighbors, and so on. Without loss of generality, the neighborhood interaction relationships in EA can be depicted as shown in Fig.4.

It is desirable that the evolutionary individual can learn from the elite neighbor's state as its next state. Essentially, according to the Q-learning, the main idea of the NDI learning is to access the $\mathrm{Q}$ values of each individual's neighbors. That is, if a 1-interval neighbor $y$ of the individual $x$ gets the maximum $Q$ value, then this individual $x$ will be transmitted to the state of $y$ at the next evolutionary time step.

It is desired that an individual at the state $s^{t}$ can receive a reward $r_{s^{t}}$ immediately and then it moves to the state $s^{t+1}$ of its 1 -interval neighbors according to an optimal strategy $s y$. Then under this optimal strategy, in order to maximize the total discounted expected reward, the Q-learning function can be formulated as

$$
Q_{t}\left(s^{t}\right)=\left(1-\alpha_{t}\right) Q_{t-1}\left(s^{t}\right)+\alpha_{t}\left[r_{t}+\gamma \max _{s^{1-\text { int }}} Q\left(s^{1-\text { int }}\right)\right]
$$

where 1-int represents one member of $R_{l}$, which is the set of 1-interval neighbors of this individual. Note that there is a case that each individual has its own 1-interval neighbors, which causes the computation imposed by Eq. (7) is made in an infinite loop, thus we pre-set a threshold $R$-interval to control this process. That is, when the $R$-interval neighbor is involved, its immediate reward is employed instead of the discounted expected reward value.

Finally, the NDI learning mechanism is presented in Algorithm 4.

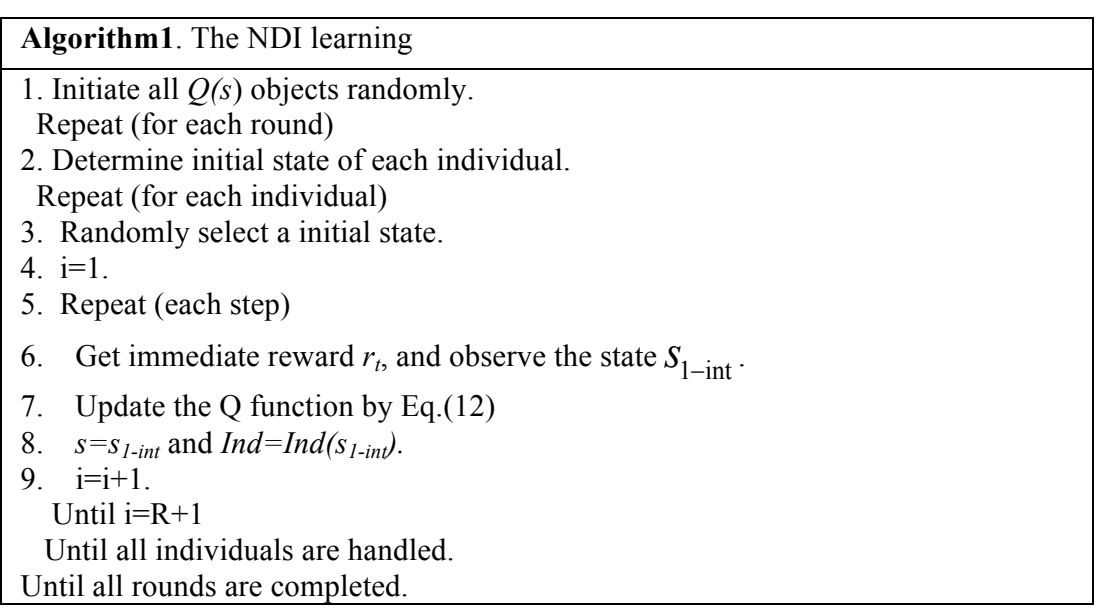

\subsubsection{Indicator-based evolution rules of population}

\section{(1) Population initialization}

For an arbitrary $D$-dimensional problem to be optimized, the spatial distribution of initial solutions in EA algorithm significantly affects the problem-solving performance during the optimization process. Intuitively, the population initialization strives to generate $N$ food sources evenly distributed in the search space, in order to significantly enhance the quality of final solutions. However, it is difficult for the conventional ABC method to generate representative initial solutions that can uniformly cover the high-dimensional search space [33], because the number or proportion of initial efficient solutions in the unit space will be lowered continuously with the increasing of the dimension.

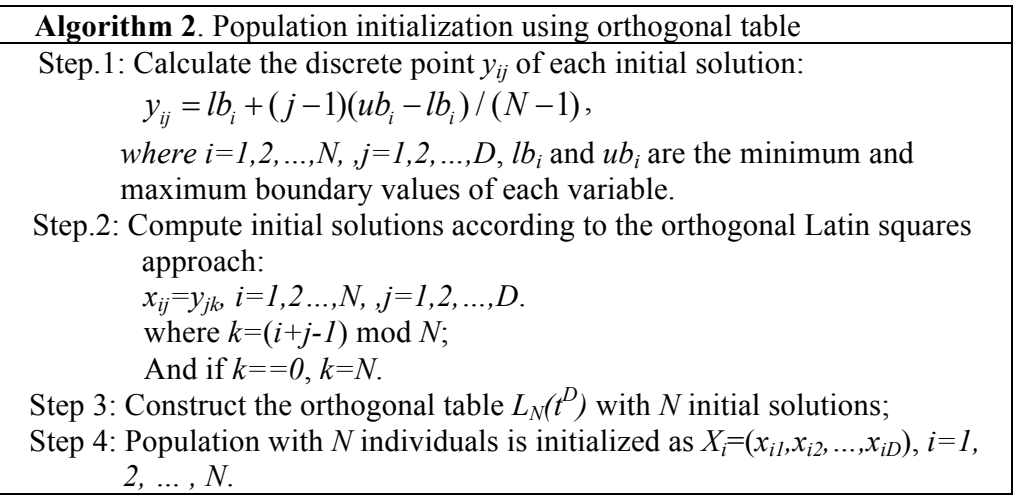

In order to address this issue, the orthogonal Latin squares approach is incorporated into population initialization in order to cover the search space with balanced dispersion and neat comparability. The orthogonal table is generated according to the principle of the orthogonal Latin squares. Suppose a population consisting of $N$ individuals (or food sources) has to be initialized in the D-dimensional search space, a orthogonal table $L_{N}\left(t^{D}\right)$ is designed deliberately where $N$ represents the number of initial solutions or table rows, $t$ is the factor level of orthogonal table, D donates the dimension of search space or the number of orthogonal arrays. Generally, this approach has a merit of obtaining optimal space coverage by consuming comparatively less tests, which has been proved theoretically by relevant theorem of non-parametric statistics in [35]. The steps of population initialization are presented in Algorithm 2.

Then the initial solution (i.e., $\left.X_{i}=\left(x_{i 1}, x_{i 2}, \ldots, x_{i D}\right), i=1,2, \ldots, N\right)$ obtained by Algorithm 2 has promising balanced dispersion and neat comparability.

\section{(2) Sending Employed Bees}

In this phase, an employed bee associated to each food source $x_{i}$ strives to explore a new temporary food source by learning from a specific neighbor, which is determined by the NDI mechanism (i.e., Algorithm 1).

In the original $\mathrm{ABC}$, given a food source $x_{i}$, its temporary position is computed as

$$
v_{i, j}=x_{i, j}+\phi\left(x_{k, j}-x_{i, j}\right)
$$

where $v_{i}$ is the newly produced position by individual $i, k$ is a randomly-selected neighbor index which are not equal to $i, j$ is a randomly-selected dimension, $\phi$ is a random coefficient within range of $[0,1]$.

As shown in Eq. (14), this operator is much like a blind mutation that only generates a single-dimensional learning from a neighbor individual, which essentially restricts information exchanging to a narrow local scope. This inevitably causes a gradually stagnation of evolution and a slow convergence to the exploration when the individual is trapped into local optima [36]. Thus, in our algorithm, unlike original ABC's inefficient search, the modified exploration equation incorporates a novel information exchanging rule based on comprehensive learning to enhance the exploration search. Exactly, to gain efficient information exchanging, each employed bee fully takes advantage of information of the elite (i.e., globally optimal solution, gbest) and its neighbor experience to drive individual-level searching and population-level evolution towards optimal status. This modified formula for employed bee is defined as

$$
v_{i, j}=x_{i, j}+\phi_{1}\left(x_{\text {gbest }, j}-x_{i, j}\right)+\phi_{2}\left(x_{k, j}-x_{i, j}\right)
$$

where $x_{\text {gbest }}$ is the global best individual from current population, $\phi_{1}$ and $\phi_{1}$ are adjustable coefficient within the scope of $[0,1]$, which usually are set to 0.5 . It is intuitive that the learning from gbest drives new candidate solution towards the global optima, as well as the conventional $k$-neighbor item enhances the in formation diversity. This also helps to avoid algorithm to be trapped into optim al position.

After a new temporary food source is yielded, its fitness is then compared against the old one with greedy approach. That is, the better one is selected as an explored food source and accordingly adjust the record of trial for this food source, namely the trial is set to zero if the new temporary one is better than the old one, otherwise, it is increased by one. This fitness assignment is restructured by $I_{s+}$ indicator [37], which maps the Pareto-optimal approximation to a real value. Formally, the formula of the fitness is given as

$$
\begin{aligned}
& F_{I \varepsilon+}(i)=\sum-e^{-I_{\varepsilon+}(\mathrm{j}-i) /(c-s)} \\
& I_{\varepsilon+}(i, \mathrm{j})=\max _{\varepsilon \in P}\left\{\varepsilon \mid \varepsilon=i_{m}-j_{m}, 1 \leq m \leq M\right\}
\end{aligned}
$$

where $i$ and $j$ denote the indexes corresponding to food sources, $\mathrm{M}$ is the number of objectives, $F_{I \varepsilon+}(i)$ is used as the fitness of a food source, $c=\max _{i, j \in p}\left|I_{\varepsilon+}(i-j)\right|$ and $s$ is a zoom factor. Essentially, $F_{I \varepsilon+}(i)$ represents the loss in quality if $i$ is deleted. Especially, this binary quality indicator $I_{\varepsilon+}$ is an enhanced extension of the Pareto dominance relation, and it donates the minimum distance by which a Pareto-optimal approximation needs to be transformed or can be transformed in each dimension in the decision space such that another approximation is weakly dominated. Thus this indicator can be directly used as dominance preserving in the multi-objective optimization. The operation procedure of employed bees is given in Algorithm 3.

\begin{tabular}{|l}
\hline Algorithm3. Sending Employed Bees \\
For $i=1$ to $N$ \\
Step1 Calculate the indicator-based fitness of each individual in population \\
by Eq.(12): \\
Step2 Choose the global best individual $x_{\text {gbest }}$ from current population. \\
Step3 Select one random dimension $j$ and select a neighbor $k$ by NDI \\
(Algorithm 1$)$ from the population \\
$\qquad v_{i, j}=x_{i, j}+\phi_{1}\left(x_{\text {gbest }, j}-x_{i, j}\right)+\phi_{2}\left(x_{k, j}-x_{i, j}\right)$ \\
Step.4: If $\left(\mathrm{F}_{\mathrm{I}}\left(v_{i}\right)>\mathrm{F}_{\mathrm{I}}\left(x_{i}\right)\right)$ \\
Add $v_{i}$ into the external archive. \\
$x_{i}=v_{i,}$ trial $l_{i}=0$ \\
Else trial \\
End IF \\
End For
\end{tabular}




\section{(3) Sending Onlooker Bees}

After finishing their exploration process, the employed bees share the food information regarding nectar amount and position with the onlooker bees by the dancing approach. Then, each onlooker bee determines a food source to exploit according to the selection probability based on the indicator-based fitness. This selection probability $\operatorname{prob}_{i}$ for the $i^{\text {th }}$ individual is defined as

$$
\operatorname{prob}_{i}=1-f i t\left(x_{i}\right) / \sum_{j=1}^{N} f i t\left(x_{j}\right)
$$

where $f i t\left(x_{i}\right)$ represents the indicator-based fitness value of $x_{i}, N$ is the population size. As shown in this formula, a better food source (i.e., with lower fitness value) is more likely to be chosen by a onlooker bee.

The mutation operation and fitness evaluation of the onlooker bee follow those of the employed bee. The operation procedure of employed bees is presented in Algorithm 4.

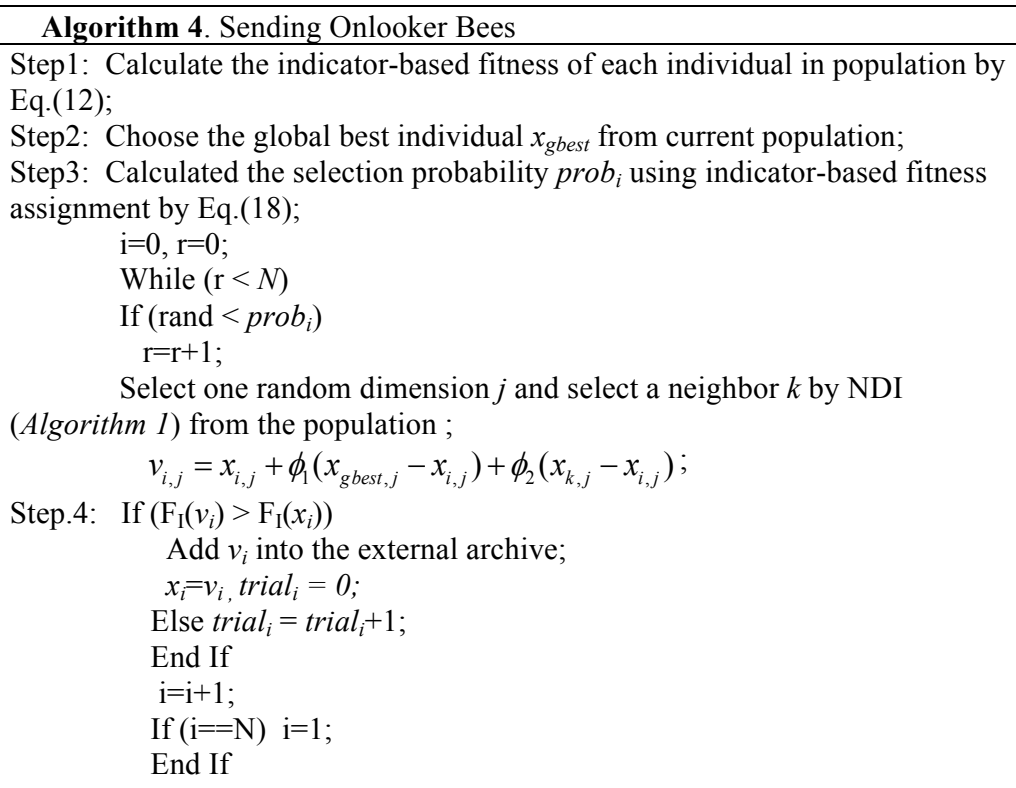

End While

\section{(4) Sending Scout Bees}

Once a food source is exhausted or cannot be improved in a limited number of cycles, the corresponding employed bee would become a scout bee, and its food source is re-initialized in the same manner as that in the original $\mathrm{ABC}$ initialization phase [30]. The algorithm is presented in Algorithm 5.

\begin{tabular}{|l|}
\hline Algorithm 5. Sending Scout Bees \\
\hline For $\mathrm{i}=1: \mathrm{N}$ \\
$\mathrm{If}\left(\right.$ trial $_{i}>$ Limit $)$ \\
For $\mathrm{j}=1: D$ \\
$\quad x_{i j}=l b_{j}+\operatorname{rand}\left(u b_{j}-l b_{j}\right)$ \\
End For \\
trial $l_{i}=0$ \\
End If \\
End For \\
\hline
\end{tabular}

\subsubsection{Archive Maintenance}

An indicator-based external population (i.e., archive) is used to curate and maintain salient non-dominated solutions. In multi-objective optimization, it is desired that an algorithm finds good approximation to Pareto-optimal front (PF) with uniformly spread of solutions as well as good convergence characteristic. Traditionally, the crowding-distance estimation [35] is used to keep diversity preservation. Specifically, given a solution, the density of solutions surrounding it is estimated as follows:

$$
C_{\mathrm{i}}=\sum_{j=1}^{M}\left(\left|f_{i+1, j}-f_{i-1, j}\right|\right)
$$

where $C_{i}$ donates the crowding distance of individual $i, M$ is the number of objective functions, $f_{i, j}$ is the $j$-th objective function value of individual $i$. And then the sum of obtained Euclidean distance values corresponding to each objective is computed as the final crowding distance value assigned to the given solution. Then the crowded comparison is implemented to compare all population members for driving the external archive evolution toward the ideal PF [36]. However, with the increasing number of the objective functions, the non-dominated solutions tend to occupy all of population space, which will inevitably make it more difficult to identify the difference between individuals. In addition, in high dimensional spaces, it is not easy to calculate the similarity between individuals. In this case, the crowding-distance estimation would unexpectedly delete significant individuals in dense regions at one time, damaging the spread characteristic of PF [37, 38].

In our algorithm, a novel dynamical indicator-based crowded comparison (DICC) mechanism is developed based on indicator-based calculation. Specifically, given a solution $x_{i}$, the indicator-based crowded metric of solutions surrounding it is re-defined as

$$
C I_{\mathrm{i}}=\sum_{j=1}^{M}\left(\mid F_{I \varepsilon+}\left(x_{i+1, j}\right)-F_{I \varepsilon+}\left(x_{i-1, j}\right)\right)
$$

where $F_{I \varepsilon+}\left(x_{i}\right)$ is the indicator-based fitness of individual $i$, which is defined as

$$
F_{I \varepsilon+}\left(x_{i}\right)=\sum_{x_{i} \in P}-e^{-I_{\varepsilon+}\left(x_{j}-x_{i}\right) /(c-s)} \text {, where } c=\max _{x_{i}, x_{j} \in p}\left|I_{\varepsilon+}\left(x_{j}-x_{i}\right)\right|
$$

It can be observed from Eq.(18) that this crowded metric to evaluate given solution is more informative by synthetically incorporating the spatial Euclidean distance and the logical performance improvement indicator. Finally, the detailed procedures of DICC are given in Algorithm 6.

\begin{tabular}{|l|}
\hline Algorithm 6. The DICC method \\
\hline Step1: Initialize the crowding distance of individuals in the non- \\
dominated population, i.e., $C I_{i}=0$; \\
Step2: Sort the individuals by computing each objective function; \\
The boundary individuals are pre-set to an infinite value to \\
ensure the availability in next selection process. \\
Step3: Compute the indicator-based crowding distance of the \\
individuals in the non-dominated archive by Eq. (19); \\
Step4: Determine the minimum individual called $I D$ in the population, \\
then delete it; \\
Step5: Re-compute the crowding distance of the individual $I D+l$ and \\
$I D-1$ by following equations respectively: \\
$\qquad C_{\mathrm{ID}+1}=\sum_{j=1}^{M}\left(\left|f_{\mathrm{ID}+2, j}-f_{\mathrm{ID}-1, j}\right|\right)$ \\
$\qquad C_{\mathrm{Flag}-1}=\sum_{j=1}^{M}\left(\left|f_{\text {Flag }+1, j}-f_{\text {Flag-2,j }}\right|\right)$ \\
Step6: If the archive size still exceeds the maximum pre-set size, $\mathrm{r}$ \\
eturn to Step3; otherwise, terminate it.
\end{tabular}

In the proposed approach, we sort obtained non-dominated solutions according to fitness evaluation, and then calculate their combined-evaluation crowded values by Eq.(20). When the number of archive members exceeds the pre-set AchiveSize, the individual with the smallest crowded fitness is deleted and the crowded fitness of remainder individuals of current population is recalculated. And then, only two neighbor individuals' crowded distances are recalculated for next loop. From Step 4 and 5 in Algorithm 6, it is apparent that this approach can avoid removing too many individuals in one region and distribute the solutions uniformly. Moreover, only two solutions are involved to perform re-calculation of the proposed crowding distance in each deleting operation, which significantly reduces the computational complexity.

\begin{tabular}{|l|}
\hline Algorithm 7. The H-MOABC algorithm \\
\hline Step1: Set relevant parameters, including Limit, Archsize, $N$ \\
$D$ \\
Step2: Initialize external archive \\
While (Termination condition is not satisfied) \\
Step3: Population Initialization() \\
Step4: Sending Employed Bees () \\
Step5: Sending Onlooker Bees () \\
Step6: Sending Scout Bees () \\
Step7: Archive Maintenance() \\
End While \\
Step8: Output archive \\
\hline
\end{tabular}

\subsubsection{The procedures of $\mathrm{H}-\mathrm{MOABC}$}

We show the H-MOABC in Algorithm 7, which incorporates the above algorithms

\subsubsection{Binarization of $\mathrm{ABC}$ algorithm}

- Vector binarization

In order to cope with the continuous type of objective function, the continuous solution vector of $\mathrm{ABC}$ has to be binarized and associated with a discrete food source [42]. Then, a fast binarization approach inspired from the method in $[42,43]$ is developed for $\mathrm{ABC}$ as below.

$$
x_{-} b_{i}=\operatorname{round}\left(\left|x_{-} c_{i} \bmod 2\right|\right) \bmod 2
$$

where $x_{-} c_{i}$ is the continuous type of solution vector and $x_{-} b_{i}$ is the binary type of vector converted from $x c_{i}$. The main motivation of this bit conversion operation lies in that continuous $x c_{i}$ is mathematically converted by double mod, which ensures its absolute value to be rounded to a binary number (i.e., 0 or 1).

\section{- Reader elimination detection}

After generating a new solution, the binary optimizer uses the reader elimination detection operation to detect whether the number of readers of current solution is less than its older solution without changing the tag coverage. Then, the vector with less readers is remained.

As for the algorithmic operations, the binary ABC (BABC) adopts the same foraging rues as those of original $\mathrm{ABC}$ in [33]. The only difference between those two algorithms is that the solution vector of binary $A B C$ should be binarized before population initialization. The working process of binary $\mathrm{ABC}$ is shown as Algorithm 8. 


\begin{tabular}{l} 
Algorithm 8. BABC algorithm \\
\hline Step1: Initialize the discrete population by Eq.(22). \\
Step2: According to [33], implement employed bees operation, \\
onlooker bees operation, scout bees operation. \\
Step 3: Reader elimination detection: \\
Calculate the reader number of current solution by sum \\
the number of state '1'. \\
$\quad$ Justify whether its tag coverage is not worse to that of \\
the old solution. \\
$\quad$ If the reader number of new solution is less than the \\
old solution, remain the new solution. Otherwise, remain the \\
old one. \\
Step 4: Justify whether the termination condition is not \\
satisfied.
\end{tabular}

\subsection{Benchmark Test}

\subsubsection{Experimental Configurations}

\section{(1) Test Problems and Performance Measures}

Six representative multi-objective benchmarks are selected to evaluate the performance of the proposed algorithm. The first six bi-objective instances include four ZDT benchmarks (i.e., ZDT1, ZDT2, ZDT3 and ZDT6) [44] and two CEC 2009 benchmarks (i.e., U1, and U2) [45]. The next tri-objective instances consist of three DTLZ instances (i.e., DTLZ1, DTLZ2 and DTLZ6) [46] and three CEC 2009 benchmarks (U8, U9, and U10) .Due to page limitation, detailed formulas of these test instances are not provided and those can be referred to $[44,45,46]$. In order to assess performance of our proposed algorithm, two performance metrics are adopted: 1) convergence metric $\Upsilon$ - IGD metric [45]; 2) spread metric $\Delta$ [38]. The further information regarding these two performance metrics can be found in $[45,38]$.

\section{(2) Algorithmic Configurations}

The H-MOABC is evaluated and compared with IBEA[37] NSGA-II [38] MOEA/D [47], SPEA2 [48], A-MOABC/NS [49] and MOABC [50]. For all the algorithms, the maximum function evaluation number is 50000. For U1 and U2 instances, the population sizes are set to 120 , for ZDTs and DTLZs, the population is 100 . Specific parameters of IBEA, MOEA/D, SPEA2 and NSGAII respectively follow those of their original references [37, 38, 47, 48]. As advised in [37], the $\kappa$ for IBEA is set to 0.05 , the recombination probability is set to 0.5 and the probability for mutation is set to 0.8 . As claimed in [38] for NSGA-II, the simulated binary crossover and polynomial crossover are used, the related crossover and mutation probability are set to $p c=0.9$ and $p m=1 / D$ respectively where $D$ donates the number of decision variables. The distribution indices for crossover and mutation are set to $\eta_{c}=\eta_{\mathrm{m}}=20$ respectively. The simulated binary crossover and polynomial mutation are employed with typical parameters as suggested in [47], the number of weight vectors is the same as its population size, and the neighborhood size is initialized with 20. For SPEA2, the SBX and polynomial mutation are employed to yield the offspring for SPEA2, and other parameters of SPEA2 can refer to [48]. For the proposed HMOABC, a reasonable set of parameter values are used empirically as following: Archive is equal to the population size, and Limit $=200$. Note that our experiments mainly focus on the empirical parameter configure, yet the best parameter configuration will be investigated further in future work.

\subsubsection{Results and Analysis}

The computational results of involved algorithms over bi-objective and triobjective benchmarks are reported in Table 4, Table 5, Fig.5, Fig. 6 and Fig.7 in the supplementary material. Note that due to paper limit, these experimental results including Table 4, Table 5, Fig.5, Fig. 6 and Fig.7. are given as the format of supplementary material following this paper. In Table 4 in the supplementary material, the mean results w.r.t the averaged IGD values over all trials for each algorithm are given. For each test problem, the averaged value and standard deviation of IGD are reported, and the best result among involved algorithms is marked in bold. In order to identify the significance of performance difference between those results obtained by H-MOABC and its counterparts, the Wilcoxon test is applied to obtained results with a level of significance $a=0.05$ [51]. In the test, the two-sample test value satisfying $z>1.640$ indicates that the proposed algorithm is significantly superior to its counterparts. Conversely, $z<1.640$ implies that it is significantly worse. In the figures, both the true PF and the non-dominated solutions obtained by H-MOABC, NSGAII, and MOEA/D are explicitly plotted. Those experimental results are all based on the trial with the lowest IGD value.

Fig. 7 in the supplementary material demonstrates the evolution of IGDmetric values of one random run via the number of function evaluations in involved algorithms on these test instances. From Fig.7, it is apparent that HMOABC obtains the lowest IDG-metric values on almost all test functions, while converges faster on ZDT1, ZDT2, ZDT3, UF1, UF2, DTLZ1 and UF10. For other instances, such as ZDT6, DTLZ2, UF8 and UF9, SPEA2 and IBEA have slightly faster convergence rate than H-MOABC. Especially, on ZDT6, the indicator-based algorithm IBEA ranks the best, followed by H-MOABC. However, it is clear from Fig.7 that SPEA2 and IBEA are easy to trap into local optima. This is also the principal reason of compared algorithms could not obtain better IGD-metric values than H-MOABC on the test instances. The elaborate experimental analysis regarding these results is implemented as follows.

\section{- Comparison of NSGA-II, MOEA/D, SPEA2and IBEA}

From results of Table 4 in the supplementary material, it can be observed that $\mathrm{H}-\mathrm{MOABC}$ generally finds better results in term of averaged IGD values than NSGA-II and MOEA/D on most of bi-objective benchmarks. For instances, compared with its counterparts (i.e., NSGA-II, IBEA and MOEA/D), HMOABC obtains about a approximately one order of magnitude boost in IGD performance for ZDT1, ZDT2 and ZDT6, and over 50\% for U1. Especially, on ZDT2 and ZDT6, the H-MOABC obtains the best average ranking largely outperforming other algorithms. On ZDT3, MOEA/D performs best of all. On ZDT1 and U2, IBEA also exhibits satisfactory performance, just a little worse than H-MOABC. On the other hand, the $z$ value of two-sample Wilcoxon test shows a significant improvement of H-MOABC over NSGAII MOEA/D, IBEA and SPEA2 on five of involved test problems. From Fig. 5 in the supplementary material, the plotted contour profile of non-dominated solutions obtained by $\mathrm{H}$ MOABC looks more close to the true PF than those obtained by its counterparts, which can be verified by the $\Delta$-metric results in Table 4 . As depicted as above section, the $\Delta$-metric aims to measure the extent of solution spread among final solutions. From Table 4, we can observe that on those benchmarks including ZDT1, ZDT3, U1 and $\mathrm{U} 2$, the $\mathrm{H}-\mathrm{MOABC}$ performs more competitive to other algorithms. The noticeable performance improvement of $\mathrm{H}-\mathrm{MOABC}$ on these bi-objective benchmarks can be ascribed to the dynamical indicator-based crowded distance method incorporated in H-MOABC to maintain population diversity.

For the tri-objective test problems, the H-MOABC exhibits obvious performance improvement over its counterparts in term of IGD-metric, especially on DTLZ2, DTLZ6, U9 and U10, where the advantage is significantly identified by the Wilcoxon test. Generally, compared with biobjective problems, these tri-objective instances are more difficult to tackle for the test algorithms, whereas the H-MOABC still keeps stable searching performance in approximation and uniformity. From Table 5 in the supplementary material, it is visibly observed that H-MOABC can rank first on five benchmarks and ranks second on one benchmark in terms of IGD-metric, and obtains IGD values lower than 0.01 on DTLZ1, DTLZ2 and DTLZ6. For spread metric, H-MOABC exhibits obvious advantage that it obtains the satisfactory averaged ranking on all test problems, ranking first on four benchmarks (i.e., DTLZ1, DTLZ6, U9 and U10), ranks second on four benchmarks (i.e., DTLZ2 and U8). From Fig. 6 in the supplementary material, it can be observed that, three algorithms perform similarly on DTLZ2. For U8, NSGA-II and H-MOABC perform slightly better than IBEA, MOEA/D and SPEA2 in uniformity. For more complex U9 and U10 with discontinuous Pareto fronts, H-MOABC exhibits a better approximation other algorithms. According to these results, the indicator-based evaluation rules and the dynamical crowded distance estimation method are essentially validated.

\section{- Comparison of multi-objective ABC algorithms}

In this section, the H-MOABC is compared with A-MOABC/NS [49] and MOABC [50] on a set of multi-objective benchmark functions. A-MOABC/NS is an asynchronous multi-objective $\mathrm{ABC}$ using non-dominated sorting procedure proposed by Akay [49] and its experimental results are directly taken from its original literatures [49]. MOABC is a Pareto-based multi-objective $\mathrm{ABC}$ algorithm using external archive [50]. Due to the page limit, we have included the computational results of the algorithms in Table 6 in the supplementary material of this paper.

Table 6 shows experimental results of $\mathrm{H}-\mathrm{MOABC}, \mathrm{A}-\mathrm{MOABC} / \mathrm{NS}$ and MOABC on bi-objective U1, bi-objective U2, bi-objective U3, tri-objective U8, tri-objective U9 and tri-objective U10. It is seen from Table 6 that H-MOABC obtains satisfactory results on most of the test functions including U1, U2, U8 and U10. Meanwhile, A-MOABC also performs well on U3 and U9. On U1 and U10, H-MOABC outperforms its counterparts in terms of the mean, best and standard deviation of IGD results. On U2 and U8, H-MOABC gets the best values of the mean and best while A-MOABC obtains the best standard deviation value. On U3 and U9, H-MOABC performs somewhat laggard in the mean values. However, it still finds the best values of best and standard deviation, which verifies its performance stability.

\section{RNP SIMULATION AND COMPUTATION RESULTS}

\subsection{H-MOABC-based Implementation for Two-level RNP Model}

\subsubsection{RNP Solution Representation}

- Definition of continuous individual

The continuous decision solution in the bottom-level optimization, involving the position and radiated power range of each reader, can be encoded as shown in Table 3.

- Definition of binary individual 
The discrete decision variable in the top-level optimization as defined in Table 2 can be handled as follows:

Given an individual $i$ at time step $t$, its binarized representation can be expressed as

$$
X_{i}^{t}=\left(x_{i 1}^{t}, x_{i 2}^{t}, \ldots, x_{i N_{\max }}^{t}\right), \quad x_{i j}^{t} \in[0,1], j=1,2, \ldots, N_{\max }
$$

where $N_{\max }$ is the maximum number of readers determined by the total deployment cost of the RFID network, and each element $x_{i j}^{t}=0$ if the corresponding reader is absent, and $x_{i j}^{t}=1$ otherwise.

- Best compromise solution based on fuzzy decision

Among the set of obtained Pareto-optimal solutions, a fuzzy-based mechanism is employed to select one solution as the best compromise solution for decision maker in RFID system [52-59, 62,63]. In this mechanism, each objective function of $i$-th solution can be donated by the following function $\mu_{i}$ :

$$
\mu_{i}= \begin{cases}1 & f_{i} \leq f_{i}^{\min } \\ \frac{f_{i}^{\max }-f_{i}}{f_{i}^{\max }-f_{i}^{\min }} & f_{i}^{\min } \leq f_{i} \leq f_{i}^{\max }, \\ 0 & f_{i} \geq f_{i}^{\max }\end{cases}
$$

where $f_{i}^{\text {max }}$ and $f_{i}^{\text {min }}$ are lower and upper boundary values of $i$-th objective function respectively.

Then, for each obtained solution, the normalized $\mu^{k}$ can be calculated as:

$$
\mu^{k}=\frac{\sum_{i=1}^{N_{o b j}} \mu_{i}^{k}}{\sum_{k=1}^{M} \sum_{i=1}^{N} \mu_{i}^{k}}
$$

where $M$ is the number of obtained solutions, and $N_{o b j}$ is the number of the objective functions. The higher value of $\mu^{k}$ means the greater satisfaction of the corresponding solution.

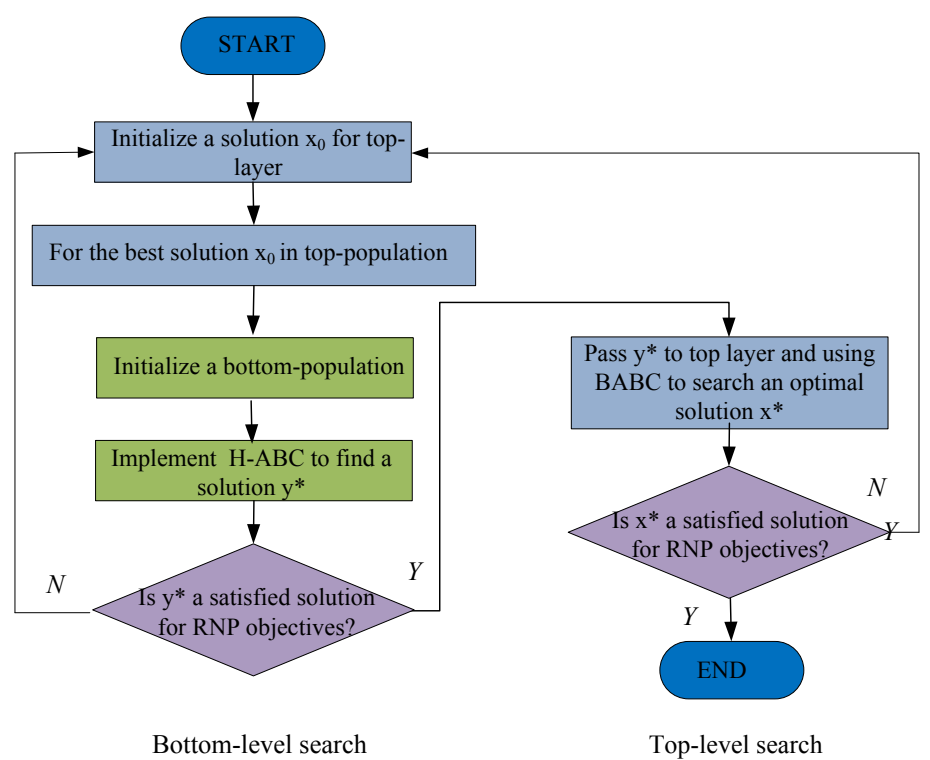

Fig. 8. The two-level RNP optimization process based on H-MOABC.

\subsubsection{Objective Function Transformation}

First, the bottom-level optimization takes Eq. (10) formulated in Section 2 as the multi-objective objectives below:

$$
F_{\text {Bottom }}\left(X_{i}^{B}\right)=\min _{X^{B}}\left\{f_{1}\left(X_{i}^{B} \mid X_{\text {best }}{ }^{T}\right), f_{2}\left(X_{i}^{B} \mid X_{\text {best }}{ }^{T}\right) \ldots, f_{4}\left(X_{i}^{B} \mid X_{\text {best }}{ }^{T}\right)\right\}
$$

where $X_{i}^{B}$ is the continuous vector of bottom-level solution $i$, which has been defined in Section 5.1.1, and $X_{j}^{T}$ is the discrete vector of top-level best solution so far, which has been defined in above section.

And then the objective function of top-level optimization derived from Eq. (8) can be listed as below:

$$
\begin{aligned}
& F_{T}\left(X_{k}^{T}(\mathrm{t}+1)\right)=w_{1} \eta\left(1-\exp \left(-\sum_{u=1}^{N_{\max }} c_{u} x_{k u}^{T}\right)\right)+w_{2}\left[\sum_{u=1}^{N_{\max }} c_{u} x_{k u}^{T}-I_{\text {max }}\right]^{+} \\
& +\mathrm{w}_{3}\left[\mathrm{f}_{1}\left(X_{k}^{T}(\mathrm{t}+1) \mid X_{\text {best }}^{B}\right)-\mathrm{f}_{1}\left(X_{k}^{T}(\mathrm{t}) \mid X_{\text {best }}^{B}\right)\right]^{+}, \quad(\sigma)^{+}= \begin{cases}\sigma & \text { if } \sigma>0 \\
0 & \text { else }\end{cases}
\end{aligned}
$$

where $w_{1}, w_{1}$ is an user-defined inertia, and empirically set to 0.5 in our experiment, $w_{2}$ and $w_{2}$ is the punishment coefficients, $X^{B}{ }_{\text {best }}$ is the obtained best decision from bottom-level optimizer. Note that the main goal of top-level optimization is to reduce redundant reader number while ensuring the optimal tag coverage, thus the $f_{l}$ function is considered in this top-level objective.

\subsubsection{H-MOABC-based Implementation for RNP}

Step 1. Initialization in the top layer. Set a binary reader-switching vector $R S=[1,1, \ldots, 1]$, which denotes that all the readers are initially deployed in the network. Initialize a solution $X_{0}^{T}=R S$ for the optimizer in the top layer. Note that each solution $X_{i}^{T}$ in the top layer denotes an advisable scheme regarding the reader number to drive a bottom-level search process.
Step 2. Deployment parameter optimization in the top layer. For each solution $X_{i}^{T}$, a bottom-level population $\left[X_{l}^{B}, \ldots, X_{i}^{B}\right]$ are randomly initialized for H-MOABC-based optimization. $X_{i}^{B}$ represents the $\mathrm{x}$-y coordinates and transmitted power level of a deployed reader.

Step 2.1. For each solution $X_{i}{ }^{B}$ in bottom-level, its fitness is calculated by Eq.(26).

Step 2.2. According to Algorithm 1-Algorithm5, three algorithmic operations including sending employed bees, sending onlooker bees and sending scout bees are implemented respectively to update the bottom-level population.

Step 2.3. The bottom-level multi-objective optimization operations are repeated until the terminal condition (i.e., the maximum function evaluation number) is met. Then send current best bottom-level solution $X_{\text {best }}{ }^{B}$ to the toplevel.

Step 3. Adjust reader number in the top layer. Based on current parameters configure (i.e., $X_{\text {best }}{ }^{B}$ ) of readers from the bottom layer, Then the binary $\mathrm{ABC}$ optimizer in the top layer strives to eliminate redundant readers from current $X_{i}^{T}$ while the tag coverage is maintained.

Step 3.1. With the bottom-level feedback $X_{\text {best }}^{B}$, the fitness of each top-level $X_{i}^{T}$ is computed by Eq. (27).

Step 3.2. Implement the binary $A B C$ paradigm to update the top-level population.

Step 3.3. The top-level optimization operations are repeated until terminal condition (i.e., the maximum iteration number) is met.

Step 4. If the terminal condition of the whole system (total maximum iteration number) is not met, return Step.2; otherwise, output the optimal solution.

The flowchart of this two-level RNP optimization process is shown in Fig. 8.

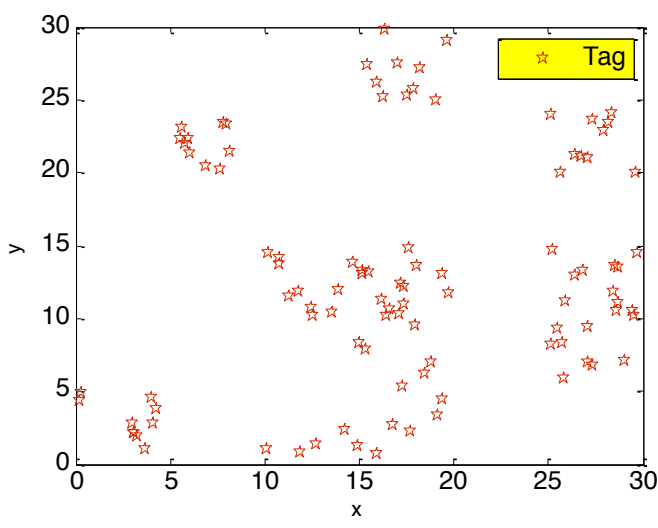

(a) $C d 100$

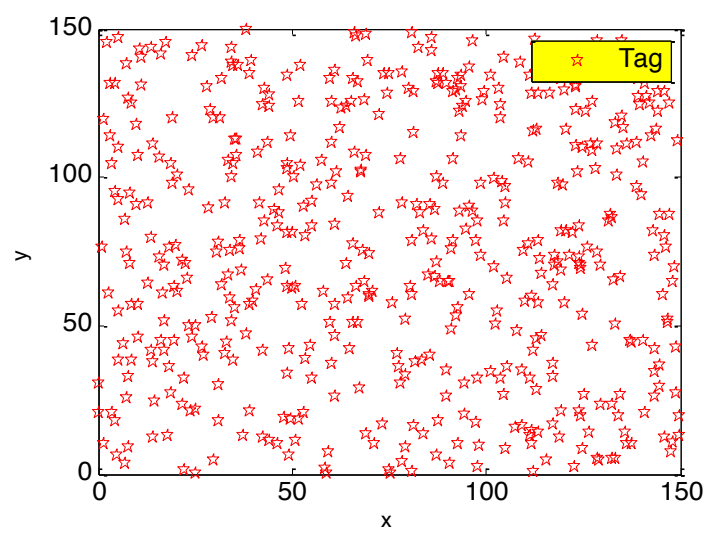

(b) $R d 500$

Fig. 9. The tag distribution in two test scenarios

\subsection{Experiment Configuration}

The mobile readers and passive tags used here are compliant with EPC Class 1 Gen 2. The proposed H-MOABC-based scheme is evaluated on two typical RNP instances, namely $C d 100$ and $R d 500$ (shown in Fig. 9). The $C d 100$ instance is simulated on a $30 \mathrm{~m} \times 30 \mathrm{~m}$ working space with 100 clustered distributed tags. The Rd500 instance is deployed in a $150 \mathrm{~m} \times 150 \mathrm{~m}$ working space with 500 randomly distributed tags. According to references [13, 24], the related RFID system parameters can be computed and listed in Table 6 . Specially, the CMOABC algorithm has been adopted and validated in multiobjective RNP optimization in previous work [13], thus it is employed for comparison in this experiment. The parameters setting for H-MOABC and NSGA-II can be same as Section .4.1 and the CMOABC parameters can be set the same as the original reference [13].

Table 6. The parameter configurations of $C d 100$ and $R d 500$

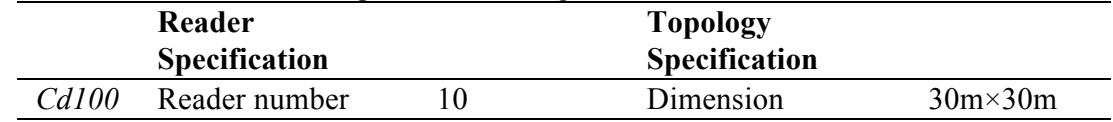




\begin{tabular}{|c|c|c|c|c|}
\hline \multirow{7}{*}{$R d 500$} & Radiated power & $0.20-3.0$ watt & Tag number & 100 \\
\hline & Interrogation range & $2-3 m$ & Tag distribution & Clustered \\
\hline & Interference range & $3.0-4.0 \mathrm{~m}$ & $\begin{array}{ll}\text { tag } & \text { power } \\
\text { threshold } & \end{array}$ & $-11.20 \mathrm{dBm}$ \\
\hline & Reader number & 50 & Dimension & $150 \mathrm{~m} \times 150 \mathrm{~m}$ \\
\hline & Radiated power & $0.20-3.0$ watt & Tag number & 500 \\
\hline & Interrogation range & $2-3 m$ & Tag distribution & Random \\
\hline & Interference range & $3.0-4.0 \mathrm{~m}$ & $\begin{array}{ll}\text { tag } & \text { power } \\
\text { threshold } & \end{array}$ & $-11.20 \mathrm{dBm}$ \\
\hline
\end{tabular}

\subsection{Computation Results with Redundant Reader Elimination}

\subsubsection{Results on $C d 100$}

\section{- Bi-objective results}

Table 7 shows experimental results by individually optimizing each singleobjective function on $C d 100$ to scientifically access the boundary points of the following trade-off curve. Then, the bi-objective Pareto-optimal solution distribution obtained by H-MOABC, CMOABC and NSGA-II are provided visually in Fig.10, where the associated bi-objective pairs include six possible instances, namely $f_{1}$ (tag coverage) $-f_{2}$ (reader interference), $f_{l}$ (tag coverage) $-f_{3}$ (economic efficiency), $f_{l}$ (tag coverage) $f_{4}$ (load balance), $f_{2}$ (reader interference) $-f_{3}$ (economic efficiency), $f_{2}$ (reader interference)- $f_{4}$ (load balance), and $f_{3}$ (economic efficiency) $f_{4}$ (load balance). After that, among the set of Paretooptimal solutions, one best comprised solution should be chosen as the feasible RFID deployment scheme. Accordingly, Table 8 gives the best compromise solutions based on fuzzy-decision method (i.e., Eqs.(18) and (19)) for each biobjective optimization instance, including the optimal reader number from the top-level, each deployed reader's coordinates $(\mathrm{X}-\mathrm{Y})$ and radiated power from the bottom-level.

In the comparison process, two Pareto front properties, namely convergence and diversity, are taken into consideration. From Fig. 10, it is apparent that the Pareto-optimal solutions by H-MOABC distribute more uniformly and the corresponding approximated trade-off curve converges better than its counterparts on most cases. Specifically, CMOABC and NSGA-II cannot approximate a well converged and appropriately distributed Pareto front on $f_{l^{-}}$- $f_{3}, f_{2}-f_{3}$ and $f_{3}-f_{4}$. From Table 8 it is observed that each objective function value in the best compromise solutions is generally close to the corresponding ideal values shown in Table 7. Moreover, by embedding top-level binary optimizer, H-MOABC employs much fewer RFID readers than CMOABC and NSGA-II. These results explicitly show that $\mathrm{H}-\mathrm{MOABC}$ is characterized by remarkable global search capability through potentially covering the entire Pareto front.

\section{- Tri -objective results}

The tri-objective Pareto-optimal solution distributions obtained by $\mathrm{H}$ MOABC, CMOABC and NSGA-II under four tri-objective instances are illustrated in Fig. 11, where these associated tri-objective pairs include $f_{l}$ (tag coverage) $-f_{2}$ (reader interference) - $f_{3}$ (economic efficiency), $f_{1}$ (tag coverage) $f_{2}$ (reader interference) - $f_{4}$ (load balance), $f_{1}$ (tag coverage) $-f_{3}$ (economic efficiency) $-f_{4}$ (load balance), and $f_{2}$ (reader interference) - $f_{3}$ (economic efficiency) - $f_{4}$ (load balance). Table 9 lists the best compromise Pareto-optimal solutions for each tri-objective optimization pair.

As shown in Fig.11, the Pareto-optimal front approximated by H-MOABC exhibits significantly better both convergence and diversity than other algorithms on most tri-objective cases. Especially, for $f_{1-}-f_{2}-f_{3}, f_{1}-f_{3}-f_{4}$ and $f_{2}-f_{3^{-}}$ $f_{4}, \mathrm{H}-\mathrm{MOABC}$ finds more non-dominated solutions than its counterparts. From 8 , it can be observed that $\mathrm{H}-\mathrm{MOABC}$ can eliminate much more redundant RFID readers than other algorithms. According to the best comprise solutions obtained by each algorithm in Table 9, Fig. 12 shows the reader locations and radiated power contours for the four three-objective instances in a visible way. From these figures, we can observe that the H-MOABC algorithm is a powerful tool to solve these tri-objective RNP instances. Note that these experimental results are obtained by $\mathrm{H}-\mathrm{MOABC}$ and other algorithms in the specific tagsclustered RFID scenario, which is pre-defined as $C d 100$ by users. This does not help to validate the problem-independence of the involved algorithms. However, it is not the focus of this paper, here we do not consider the effect of the parameter configuration of the RNP instance on the performances of the test algorithms.

Table 7. Results obtained by H-MOABC, CMOABC and NSGA-II on Cd100

\begin{tabular}{|c|c|c|c|c|c|c|c|c|c|c|c|}
\hline \multicolumn{3}{|c|}{$f_{1:}$ tag coverage } & \multicolumn{3}{|c|}{$f_{2}:$ reader interference } & \multicolumn{3}{|c|}{$f_{3}:$ economic efficiency } & \multicolumn{3}{|c|}{$f_{4}:$ load balance } \\
\hline H-MOABC & CMOABC & NSGA-II & H-MOABC & CMOABC & NSGA-II & H-MOABC & CMOABC & NSGA-II & H-MOABC & CMOABC & NSGA-II \\
\hline $1.421 \mathrm{e}-7$ & $2.102 \mathrm{e}-6$ & $2.625 e-6$ & $1.212 \mathrm{e}-1$ & $4.401 \mathrm{e}-1$ & $4.889 \mathrm{e}-1$ & $2.404 \mathrm{e}-2$ & $1.112 \mathrm{e}-1$ & $3.093 \mathrm{e}-1$ & $2.031 \mathrm{e}-2$ & $5.422 \mathrm{e}-2$ & $1.213 \mathrm{e}-1$ \\
\hline
\end{tabular}

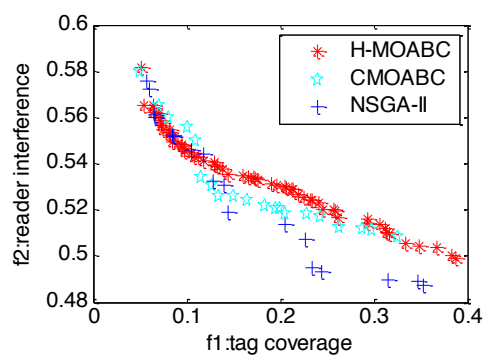

(a) $f_{1}-f_{2}$

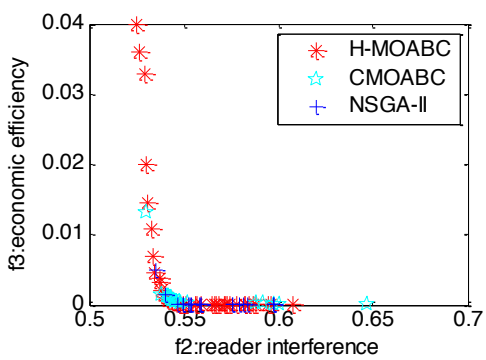

(d) $f_{2}-f_{3}$

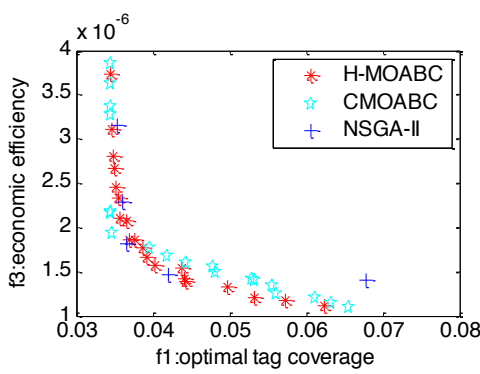

(b) $f_{l^{-}} f_{3}$

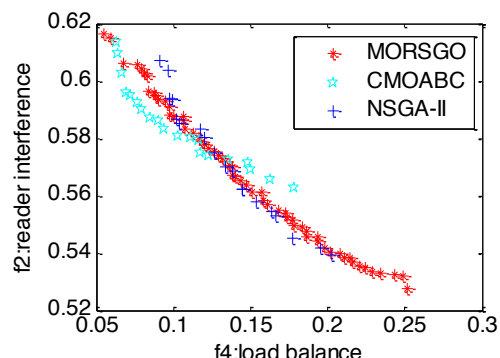

(e) $f_{2}-f_{4}$

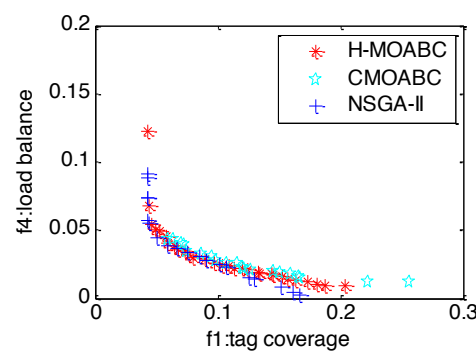

(c) $f_{1^{-}} f_{4}$

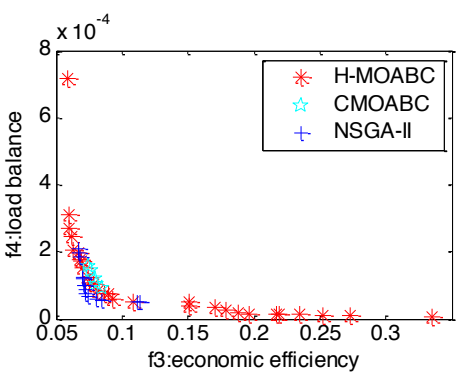

(f) $f_{3^{-}} f_{4}$

Fig. 10. Distribution of Pareto-optimal solutions obtained by H-MOABC, CMOABC and NSGA-II on (a) $f_{1}-f_{2}$, (b) $f_{1}-f_{3}$, (c) $f_{1}-f_{4}$, (d) $f_{2}-f_{3}$, (e) $f_{2}-f_{4}$, and (f) $f_{3}-f_{4}$.

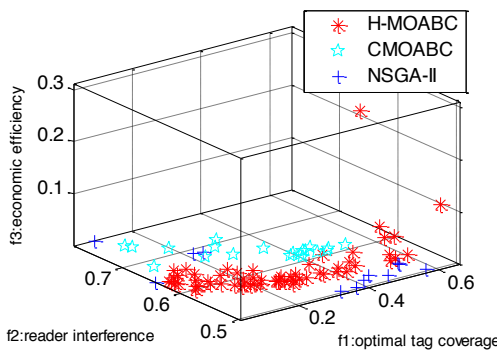

(a) $f_{1}-f_{2}-f_{3}$

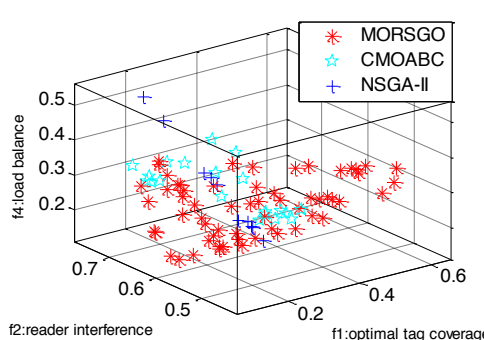

(b) $f_{1-}-f_{2}-f_{4}$

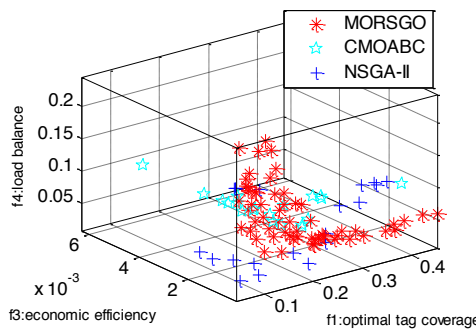

(c) $f_{1}-f_{3}-f_{4}$

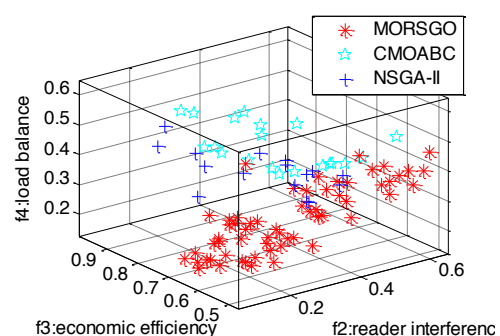

(d) $f_{2}-f_{3}-f_{4}$

Fig. 11. Tri-objective Pareto-optimal solutions obtained by H-MOABC, CMOABC and NSGA-II on (a) $f_{1}-f_{2}-f_{3}$, , (b) $f_{1}-f_{2}-f_{4},\left(\right.$ c) $f_{1}-f_{3}-f_{4}$, and (d) $f_{1}-f_{3}-f_{4}$. 


\begin{tabular}{|c|c|c|c|c|c|c|c|c|c|c|c|c|c|c|c|c|c|c|}
\hline & & $f_{1^{-}} f_{2}$ & & & $f_{1}-f_{3}$ & & & $f_{1^{-}} f_{4}$ & & & $f_{2}-f_{3}$ & & & $f_{2}-f_{4}$ & & & $\boldsymbol{f}_{3}-\boldsymbol{f}_{4}$ & \\
\hline & $\begin{array}{c}\mathrm{H}- \\
\mathrm{MOA} \\
\mathrm{BC}\end{array}$ & $\begin{array}{c}\text { CMOA } \\
\text { BC }\end{array}$ & $\begin{array}{l}\text { NSG } \\
\text { A-II }\end{array}$ & $\begin{array}{c}\mathrm{H}- \\
\mathrm{MOA} \\
\mathrm{BC} \\
\end{array}$ & $\begin{array}{c}\text { CMOA } \\
\text { BC }\end{array}$ & $\begin{array}{c}\text { NSG } \\
\text { A-II }\end{array}$ & $\begin{array}{c}\mathrm{H}- \\
\mathrm{MOA} \\
\mathrm{BC} \\
\end{array}$ & $\begin{array}{c}\text { CMOA } \\
\text { BC }\end{array}$ & $\begin{array}{l}\text { NSG } \\
\text { A-II }\end{array}$ & $\begin{array}{c}\mathrm{H}- \\
\mathrm{MOA} \\
\mathrm{BC} \\
\end{array}$ & $\begin{array}{c}\text { CMOA } \\
\text { BC }\end{array}$ & $\begin{array}{l}\text { NSG } \\
\text { A-II }\end{array}$ & $\begin{array}{c}\mathrm{H}- \\
\mathrm{MOA} \\
\mathrm{BC} \\
\end{array}$ & $\begin{array}{c}\text { CMOA } \\
\text { BC }\end{array}$ & $\begin{array}{c}\text { NSG } \\
\text { A-II }\end{array}$ & $\begin{array}{c}\mathrm{H}- \\
\mathrm{MOA} \\
\mathrm{BC} \\
\end{array}$ & $\begin{array}{c}\text { CMOA } \\
\text { BC }\end{array}$ & $\begin{array}{c}\text { NSG } \\
\text { A-II }\end{array}$ \\
\hline $\mathbf{X}_{1}$ & 26.68 & 16.64 & 15.46 & 20.47 & 13.64 & 4.55 & 9.05 & 11.88 & 19.56 & 25.30 & 13.47 & 24.11 & 24.41 & 2.66 & 25.57 & 13.29 & 18.96 & 16.94 \\
\hline$Y_{1}$ & 32.75 & 14.80 & 18.27 & 30.04 & 24.35 & 22.58 & 25.84 & 8.70 & 22.31 & 14.86 & 16.08 & 11.80 & 3.49 & 19.25 & 15.02 & 24.71 & 24.03 & 9.99 \\
\hline $\mathbf{X}_{2}$ & 17.01 & 17.35 & 25.92 & 0.02 & 4.48 & 28.97 & 24.74 & 3.92 & 19.74 & 16.72 & 11.98 & 25.36 & 14.86 & 16.83 & 14.17 & 13.03 & 7.11 & 12.96 \\
\hline$Y_{2}$ & 17.13 & 18.47 & 8.56 & 13.79 & 15.15 & 31.14 & 19.92 & 26.35 & 13.12 & 17.95 & 21.28 & 23.15 & 10.48 & 14.55 & 13.20 & 22.58 & 19.06 & 17.63 \\
\hline $\mathbf{X}_{3}$ & 10.76 & 15.02 & 15.57 & 22.68 & 28.96 & 13.38 & 15.27 & 18.66 & 12.82 & 14.69 & 22.87 & 14.83 & 24.17 & 13.70 & 14.30 & 17.51 & 12.76 & 25.68 \\
\hline $\mathbf{Y}_{3}$ & 17.57 & 16.50 & 3.77 & 20.48 & 9.82 & 24.24 & 21.19 & 23.64 & 9.07 & 14.44 & 17.87 & 22.93 & 23.10 & 15.75 & 10.46 & 2.93 & 6.78 & 27.81 \\
\hline $\mathbf{X}_{4}$ & 18.83 & 10.00 & 21.58 & 11.32 & 7.25 & 17.49 & 19.89 & 6.76 & 6.72 & 14.93 & 15.94 & 10.62 & 29.41 & 25.49 & 10.25 & 13.26 & 6.88 & 31.29 \\
\hline $\mathbf{Y}_{4}$ & 14.85 & 6.53 & 16.21 & 0.01 & 29.54 & 16.25 & 13.75 & 12.91 & 13.31 & 10.79 & 15.67 & 14.32 & 12.80 & 23.34 & 13.23 & 15.62 & 28.73 & 16.26 \\
\hline $\mathbf{X}_{5}$ & 22.36 & 14.53 & 9.81 & 18.48 & 22.03 & 7.91 & 14.79 & 16.40 & 15.86 & 33.24 & 16.71 & 16.67 & 13.43 & 9.26 & 13.22 & 24.57 & 18.51 & 28.87 \\
\hline$Y_{5}$ & 17.99 & 24.36 & 23.86 & 3.09 & 20.53 & 22.58 & 14.74 & 14.29 & 2.93 & 14.54 & 22.10 & 11.19 & 13.01 & 24.40 & 16.44 & 7.87 & 25.83 & 23.57 \\
\hline$X_{6}$ & 14.54 & 20.55 & 8.16 & 7.31 & 7.96 & 34.00 & 30.45 & 4.23 & 13.69 & 14.88 & 26.01 & 12.91 & 20.00 & 15.92 & 17.73 & 6.83 & 13.20 & 6.94 \\
\hline$Y_{6}$ & 25.06 & 7.27 & 15.68 & 9.44 & 12.84 & 25.10 & 19.30 & 28.52 & 21.05 & 36.95 & 20.40 & 26.81 & 32.13 & 13.57 & 14.63 & 18.90 & 18.14 & 30.40 \\
\hline $\mathbf{X}_{7}$ & 22.97 & 17.84 & 15.08 & 14.03 & 18.69 & 15.46 & 12.56 & 9.37 & 23.66 & 34.01 & 19.75 & 8.93 & 28.33 & 30.41 & 28.22 & 18.78 & 12.97 & 17.79 \\
\hline $\mathbf{Y}_{7}$ & 29.39 & 14.70 & 9.64 & 26.67 & 23.39 & 6.96 & 4.96 & 22.43 & 26.57 & 24.81 & 28.10 & 21.99 & 18.58 & 19.64 & 19.03 & 16.57 & 12.96 & 18.39 \\
\hline $\mathbf{X}_{8}$ & 14.47 & 14.06 & 13.03 & 2.12 & 25.84 & 0.87 & 33.22 & 26.10 & 21.05 & 21.91 & 24.30 & 20.59 & 16.55 & 18.16 & 22.03 & 11.06 & 3.14 & 19.42 \\
\hline $\mathbf{Y}_{8}$ & 14.58 & 7.93 & 1.30 & 11.76 & 6.96 & 6.35 & 20.60 & 8.14 & 17.04 & 19.29 & 17.47 & 22.94 & 12.03 & 18.23 & 0.61 & 13.38 & 13.24 & 20.51 \\
\hline$X_{9}$ & 0.04 & 22.15 & 15.28 & 26.41 & 21.83 & 17.65 & 19.09 & 16.96 & 26.74 & 19.06 & 16.55 & 24.24 & 13.79 & 21.27 & 13.22 & 3.97 & 5.75 & 8.44 \\
\hline $\mathbf{Y}_{9}$ & 0.69 & 16.24 & 16.10 & 14.99 & 6.50 & 25.37 & 14.73 & 15.34 & 26.08 & 27.34 & 17.86 & 9.55 & 18.51 & 25.36 & 15.36 & 5.16 & 23.56 & 22.84 \\
\hline$X_{1}$ & 1.23 & 13.31 & 16.00 & 14.49 & 6.27 & 31.20 & 14.29 & 0.00 & 3.24 & 13.18 & 18.63 & 4.47 & 21.39 & 19.51 & 19.20 & 14.47 & 22.14 & 15.26 \\
\hline$Y_{1}$ & 26.35 & 20.33 & 22.43 & 10.43 & 28.68 & 15.59 & 14.14 & 12.81 & 18.01 & 2.94 & 15.78 & 21.34 & 18.61 & 25.43 & 13.11 & 30.66 & 22.83 & 8.40 \\
\hline $\mathbf{P}_{1}$ & 0.00 & 30.46 & 17.18 & 33.49 & 0.00 & 0.00 & 34.12 & 8.34 & 22.73 & 34.69 & 22.73 & 31.12 & 0.00 & 33.34 & 13.70 & 19.34 & 23.82 & 10.82 \\
\hline $\mathbf{P}_{2}$ & 28.20 & 32.61 & 23.95 & 0.00 & 18.92 & 19.03 & 14.79 & 0.00 & 22.79 & 34.69 & 28.04 & 33.58 & 35.72 & 34.04 & 33.76 & 36.78 & 20.37 & 19.69 \\
\hline $\mathbf{P}_{3}$ & 0.00 & 28.68 & 0.00 & 33.49 & 18.73 & 26.79 & 1.94 & 16.00 & 12.91 & 37.99 & 22.76 & 0.00 & 0.00 & 36.90 & 31.08 & 0.00 & 0.00 & 8.55 \\
\hline $\mathbf{P}_{4}$ & 36.82 & 0.00 & 31.96 & 27.17 & 26.41 & 13.71 & 15.73 & 0.00 & 16.70 & 0.00 & 0.00 & 34.71 & 32.89 & 36.50 & 0.00 & 0.00 & 0.00 & 35.93 \\
\hline$P_{5}$ & 0.00 & 22.98 & 29.14 & 0.00 & 22.44 & 31.08 & 33.90 & 25.62 & 16.30 & 38.15 & 24.01 & 11.49 & 37.58 & 0.00 & 37.56 & 37.46 & 14.59 & 22.27 \\
\hline $\mathbf{P}_{6}$ & 35.93 & 25.38 & 0.00 & 32.87 & 23.82 & 21.54 & 35.03 & 19.12 & 16.53 & 37.87 & 25.76 & 34.22 & 36.85 & 38.33 & 37.46 & 37.22 & 26.71 & 21.42 \\
\hline $\mathbf{P}_{7}$ & 36.80 & 24.29 & 30.44 & 0.00 & 0.00 & 24.46 & 0.00 & 19.92 & 0.00 & 0.00 & 31.00 & 0.00 & 0.00 & 38.46 & 37.71 & 37.32 & 21.38 & 33.13 \\
\hline $\mathbf{P}_{8}$ & 0.00 & 25.72 & 27.19 & 27.77 & 12.42 & 0.00 & 35.06 & 21.04 & 5.86 & 38.15 & 21.60 & 33.83 & 37.44 & 0.00 & 27.14 & 0.00 & 21.14 & 0.00 \\
\hline $\mathbf{P}_{9}$ & 35.37 & 0.00 & 30.73 & 0.00 & 0.00 & 33.89 & 0.00 & 0.00 & 0.00 & 38.15 & 21.39 & 29.59 & 37.97 & 38.05 & 34.75 & 36.93 & 27.66 & 0.00 \\
\hline $\mathbf{P}_{1}$ & 36.17 & 32.72 & 30.41 & 33.49 & 15.94 & 31.95 & 35.03 & 25.16 & 15.24 & 35.03 & 19.90 & 25.04 & 34.13 & 0.00 & 25.57 & 0.00 & 26.90 & 21.33 \\
\hline$f_{1}$ & $2.24 \mathrm{e}-$ & $3.43 \mathrm{e}-6$ & $7.55 \mathrm{e}$ & $2.77 \mathrm{e}-$ & $2.99 \mathrm{e}-6$ & $3.94 \mathrm{e}$ & $6.21 \mathrm{e}-$ & $6.334 \mathrm{e}-$ & $9.71 \mathrm{e}$ & $N A$ & NA & $N A$ & $N A$ & $N A$ & $N A$ & $N A$ & $N A$ & $N A$ \\
\hline$f_{2}$ & 0.42 & 0.65 & $5.75 \mathrm{E}$ & $N A$ & $N A$ & $N A$ & $N A$ & $N A$ & $N A$ & $4.51 \mathrm{e}-$ & $4.81 \mathrm{e}-1$ & $4.80 \mathrm{e}$ & $4.72 \mathrm{e}-$ & $4.21 \mathrm{e}-1$ & $6.44 \mathrm{e}$ & $N A$ & $N A$ & $N A$ \\
\hline$f_{3}$ & NA & NA & NA & $1.32 \mathrm{e}-$ & $1.41 \mathrm{e}-2$ & $1.53 \mathrm{e}$ & $N A$ & $N A$ & $N A$ & $1.13 \mathrm{e}-$ & $1.43 \mathrm{e}-2$ & $1.52 \mathrm{e}$ & $N A$ & $N A$ & $N A$ & $1.18 \mathrm{e}-$ & $1.19 \mathrm{e}-2$ & $2.10 \mathrm{e}$ \\
\hline$f_{4}$ & NA & NA & $N A$ & $N A$ & $N A$ & $N A$ & $2.64 \mathrm{e}-$ & $1.02 \mathrm{e}-1$ & $6.11 \mathrm{e}$ & $N A$ & $N A$ & $N A$ & $9.13 \mathrm{e}-$ & $1.71 \mathrm{e}-1$ & $2.36 \mathrm{e}$ & $1.41 \mathrm{e}-$ & $1.66 \mathrm{e}-2$ & $4.18 \mathrm{e}$ \\
\hline$N$ & 6 & 8 & 8 & 7 & 7 & 8 & 8 & 6 & 8 & 8 & 9 & 8 & 7 & 7 & 8 & 6 & 8 & 8 \\
\hline
\end{tabular}

Table 9. The best compromise solutions for each tri-objective pair from the Pareto front based on involved test algorithms

\begin{tabular}{|c|c|c|c|c|c|c|c|c|c|c|c|c|}
\hline \multirow[b]{3}{*}{$\mathrm{X}_{1}$} & \multicolumn{3}{|c|}{$f_{1}-f_{2}-f_{3}$} & \multicolumn{3}{|c|}{$f_{1}-f_{2^{-}} f_{4}$} & \multicolumn{3}{|c|}{$f_{I^{-}} f_{3^{-}} f_{4}$} & \multicolumn{3}{|c|}{$f_{2^{-}} \boldsymbol{f}_{3^{-}} \boldsymbol{f}_{4}$} \\
\hline & H-MOABC & CMOABC & NSGA-II & H-MOABC & CMOABC & NSGA-II & H-MOABC & CMOABC & NSGA-II & H-MOABC & CMOABC & NSGA-II \\
\hline & 18.48 & 25.04 & 22.88 & 15.17 & 26.00 & 3.60 & 20.89 & 4.01 & 16.39 & 2.74 & 24.66 & 25.05 \\
\hline $\mathbf{Y}_{1}$ & 9.54 & 21.36 & 14.60 & 13.04 & 15.71 & 6.00 & 13.09 & 6.28 & 14.06 & 4.12 & 15.73 & 16.20 \\
\hline $\mathbf{X}_{2}$ & 14.32 & 8.33 & 15.97 & 21.58 & 19.49 & 19.22 & 33.23 & 19.37 & 17.73 & 7.24 & 12.07 & 4.40 \\
\hline $\mathbf{Y}_{2}$ & 28.16 & 14.40 & 0.20 & 30.83 & 17.55 & 9.61 & 25.40 & 9.75 & 3.18 & 27.08 & 18.41 & 23.42 \\
\hline $\mathbf{X}_{3}$ & 24.14 & 8.80 & 2.25 & 12.79 & 16.85 & 9.33 & 31.45 & 17.15 & 30 & 30 & 14.60 & 25.63 \\
\hline $\mathbf{Y}_{3}$ & 8.96 & 16.50 & 27.05 & 0.48 & 14.32 & 19.66 & 6.73 & 18.85 & 16.78 & 18.95 & 15.48 & 13.17 \\
\hline $\mathbf{X}_{4}$ & 23.97 & 12.60 & 20.06 & 17.50 & 15.66 & 21.06 & 23.79 & 14.61 & 11.17 & 19.64 & 14.14 & 14.17 \\
\hline $\mathbf{Y}_{4}$ & 15.40 & 2.30 & 7.69 & 32.97 & 31.75 & 20.19 & 15.61 & 26.54 & 9.67 & 17.16 & 24.28 & 30 \\
\hline $\mathbf{X}_{5}$ & 14.16 & 8.48 & 33.06 & 21.46 & 31.75 & 10.10 & 36.62 & 15.83 & 11.58 & 13.97 & 17.56 & 15.63 \\
\hline$Y_{5}$ & 1.92 & 19.12 & 18.22 & 9.20 & 9.77 & 21.37 & 12.80 & 16.11 & 28.21 & 17.43 & 17.95 & 27.83 \\
\hline $\mathbf{X}_{6}$ & 1.62 & 7.36 & 20.35 & 5.77 & 11.54 & 11.54 & 3.51 & 12.08 & 5.66 & 21.64 & 12.71 & 19.50 \\
\hline $\mathbf{Y}_{6}$ & 11.05 & 0.11 & 23.26 & 27.28 & 3.66 & 14.04 & 27.75 & 31.90 & 25.55 & 13.16 & 16.43 & 23.33 \\
\hline $\mathbf{X}_{7}$ & 5.40 & 5.40 & 21.05 & 4.80 & 8.74 & 31.14 & 17.56 & 10.87 & 21.58 & 18.78 & 24.16 & 15.84 \\
\hline $\mathbf{Y}_{7}$ & 9.50 & 25.72 & 16.96 & 3.63 & 14.92 & 16.61 & 17.02 & 12.93 & 21.53 & 3.41 & 26.55 & 15.77 \\
\hline $\mathbf{X}_{8}$ & 24.66 & 12.54 & 5.63 & 32.63 & 22.29 & 6.50 & 4.12 & 21.80 & 32.75 & 20.81 & 21.79 & 24.99 \\
\hline $\mathbf{Y}_{8}$ & 7.35 & 7.61 & 18.95 & 21.03 & 14.73 & 33.30 & 3.54 & 20.53 & 19.77 & 30 & 7.06 & 16.89 \\
\hline $\mathbf{X}_{9}$ & 18.64 & 12.58 & 15.23 & 24.46 & 9.44 & 10.88 & 15.08 & 17.47 & 5.39 & 13.60 & 16.15 & 20.39 \\
\hline $\mathbf{Y}_{9}$ & 23.38 & 1.37 & 15.99 & 9.04 & 9.62 & 18.67 & 22.93 & 10.92 & 21.07 & 11.16 & 9.90 & 4.90 \\
\hline$X_{10}$ & 7.37 & 22.94 & 20.29 & 16.75 & 20.11 & 21.43 & 14.21 & 13.96 & 2.58 & 23.21 & 9.37 & 11.69 \\
\hline$Y_{10}$ & 0.68 & 19.29 & 3.91 & 6.58 & 16.08 & 9.67 & 1.18 & 11.50 & 11.09 & 6.96 & 8.94 & 5.62 \\
\hline $\mathbf{P}_{1}$ & 25.90 & 10.73 & 10.79 & 26.40 & 21.19 & 8.63 & 22.90 & 19.05 & 4.83 & 26.44 & 12.17 & 8.08 \\
\hline $\mathbf{P}_{2}$ & 0.00 & 0.00 & 15.23 & 0.00 & 19.95 & 9.91 & 26.46 & 14.46 & 18.49 & 22.85 & 20.19 & 7.22 \\
\hline $\mathbf{P}_{3}$ & 0.00 & 0.00 & 9.95 & 0.00 & 13.19 & 20.02 & 0.00 & 18.77 & 4.74 & 26.46 & 15.80 & 17.55 \\
\hline $\mathbf{P}_{4}$ & 25.89 & 8.26 & 14.56 & 26.40 & 15.30 & 0.00 & 21.81 & 10.89 & 21.55 & 0.00 & 14.31 & 9.48 \\
\hline $\mathbf{P}_{5}$ & 26.07 & 15.75 & 0.00 & 22.59 & 10.18 & 5.31 & 0.00 & 11.78 & 0.00 & 0.00 & 15.40 & 0.00 \\
\hline $\mathbf{P}_{6}$ & 0.00 & 21.14 & 18.89 & 20.17 & 14.88 & 3.81 & 26.18 & 10.98 & 5.58 & 0.00 & 15.56 & 17.70 \\
\hline $\mathbf{P}_{7}$ & 29.46 & 3.69 & 21.77 & 29.46 & 14.40 & 22.37 & 29.34 & 12.06 & 16.36 & 29.44 & 20.04 & 12.38 \\
\hline $\mathbf{P}_{8}$ & 29.95 & 17.79 & 29.36 & 27.92 & 0.00 & 15.07 & 22.11 & 14.92 & 23.96 & 29.65 & 24.66 & 17.95 \\
\hline $\mathbf{P}_{9}$ & 27.06 & 23.03 & 25.09 & 0.00 & 0.00 & 25.83 & 2.95 & 12.54 & 13.12 & 26.45 & 15.73 & 0.00 \\
\hline$P_{10}$ & 0 & 20.34 & 26.15 & 30 & 14.66 & 16.74 & 20.89 & 0.00 & 16.39 & 27.38 & 12.07 & 24.34 \\
\hline$f_{1}$ & $2.38 \mathrm{e}-7$ & $2.31 \mathrm{e}-6$ & $5.36 \mathrm{e}-7$ & $5.46 \mathrm{e}-6$ & $5.49 \mathrm{e}-6$ & $4.38 \mathrm{e}-6$ & $6.93 e-6$ & $5.43 \mathrm{e}-6$ & $3.76 \mathrm{e}-5$ & $N A$ & $N A$ & $N A$ \\
\hline$f_{2}$ & $5.25 \mathrm{e}-1$ & $3.46 \mathrm{e}-1$ & $5.22 \mathrm{e}-1$ & $5.31 \mathrm{e}-1$ & $5.18 \mathrm{e}-1$ & $5.81 \mathrm{e}-1$ & $5.01 \mathrm{e}-1$ & $5.77 \mathrm{e}-1$ & $5.02 \mathrm{e}-1$ & $3.12 \mathrm{e}-1$ & $4.23 \mathrm{e}-1$ & $5.53 \mathrm{e}-1$ \\
\hline$f_{3}$ & $1.68 \mathrm{e}-2$ & $4.02 \mathrm{e}-2$ & $5.66 \mathrm{e}-2$ & $N A$ & $N A$ & $N A$ & $N A$ & $N A$ & $N A$ & $6.29 \mathrm{e}-1$ & $6.57 \mathrm{e}-1$ & $7.95 \mathrm{e}-1$ \\
\hline$f_{4}$ & $N A$ & $N A$ & $N A$ & $3.88 \mathrm{e}-1$ & $3.90 \mathrm{e}-1$ & $6.42 \mathrm{e}-1$ & $8.31 \mathrm{e}-1$ & $7.66 \mathrm{e}-1$ & $8.88 \mathrm{e}-1$ & $1.189 \mathrm{e}-1$ & $2.03 \mathrm{e}-1$ & $3.66 \mathrm{e}-1$ \\
\hline $\mathrm{Ne}$ & 6 & 8 & 9 & 7 & 8 & 9 & 7 & 9 & 8 & 7 & 8 & 8 \\
\hline
\end{tabular}

where $\mathrm{X}_{i}$ and $\mathrm{Y}_{i}$ donate the coordinates of the $i$ th reader in the working region, $N_{e}$ donates the optimal reader number and $\mathrm{P}_{i}$ is the radiate power of the $i$ th reader.

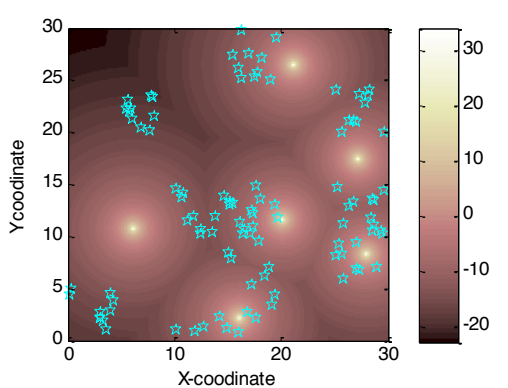

(a) H-MOABC

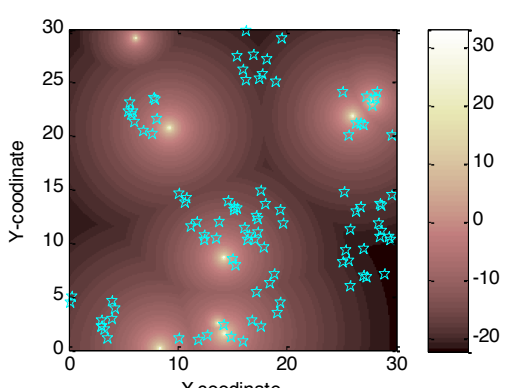

(b) CMOABC

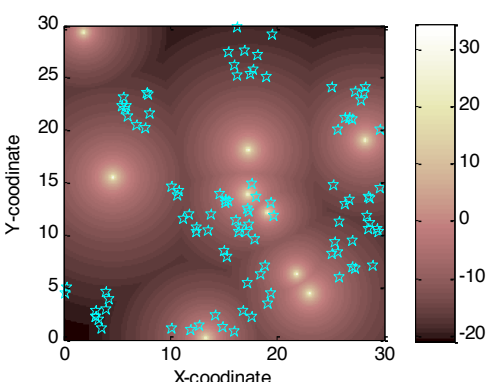

(c) NSGA-II 


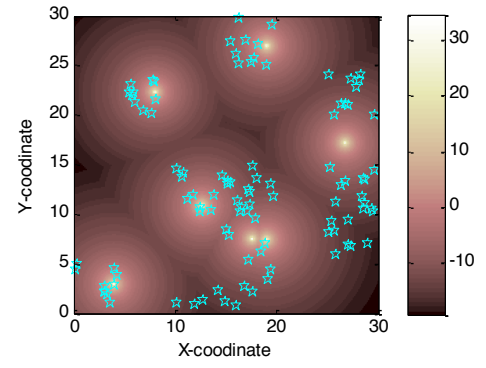

(d) H-MOABC

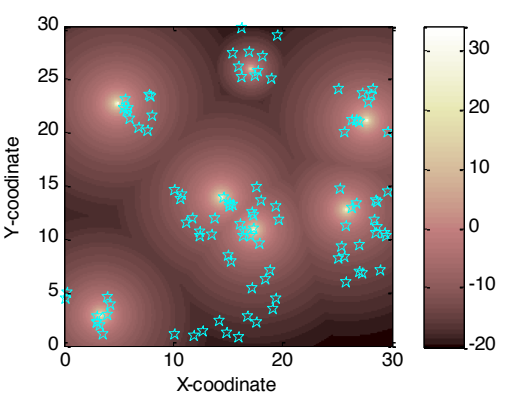

(g) H-MOABC

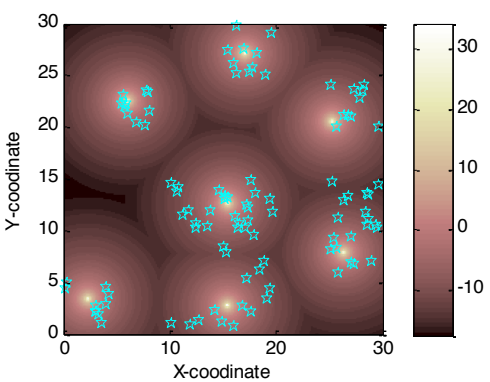

(j) H-MOABC

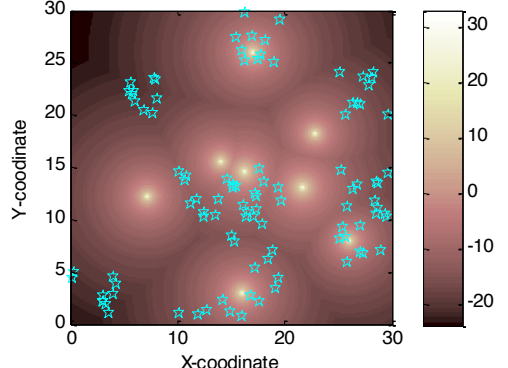

(e) $\mathrm{CMOABC}$

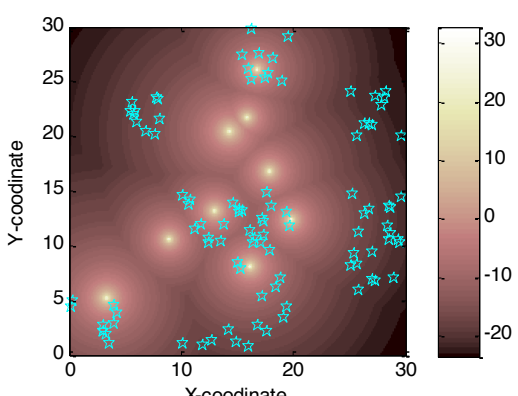

(h) $\mathrm{CMOABC}$

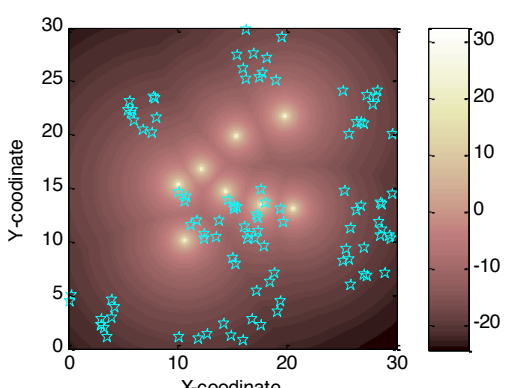

(k) $\mathrm{CMOABC}$

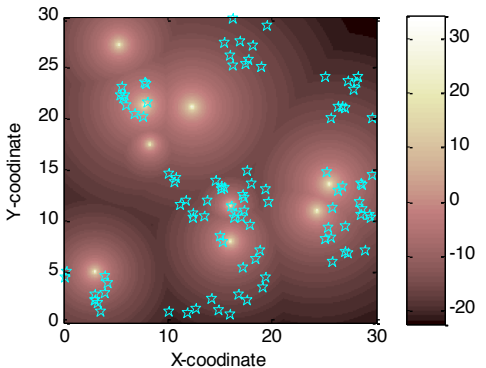

(f) NSGA-II

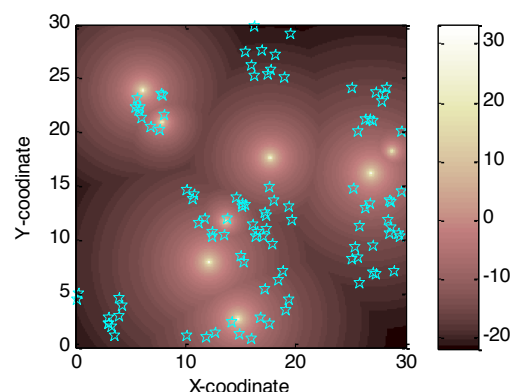

(i) NSGA-II

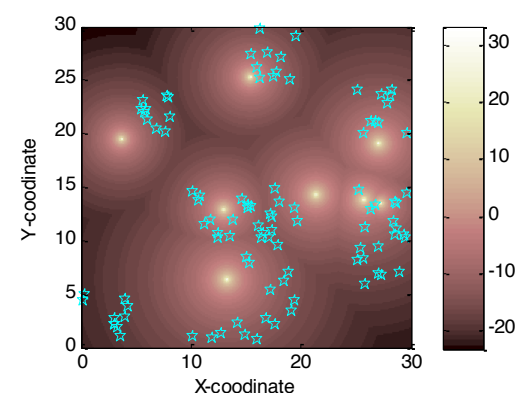

(1) NSGA-II

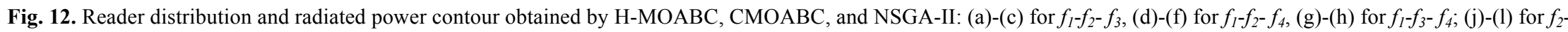
$f_{3}-f_{4}$.

Table 10. Results of each objective functions by H-MOABC, CMOABC and NSGA-II on Rd500

\begin{tabular}{|c|c|c|c|c|c|c|c|c|c|c|c|c|}
\hline & \multicolumn{3}{|c|}{$f_{1}: t a g$ coverage } & \multicolumn{3}{|c|}{$f_{2}:$ reader interference } & \multicolumn{3}{|c|}{$f_{3}:$ economic efficiency } & \multicolumn{3}{|c|}{$f_{4}:$ load balance } \\
\hline$f$ & $\begin{array}{l}\text { H-MOABC } \\
1.647 \mathrm{e}-6\end{array}$ & $\begin{array}{l}\text { CMOABC } \\
7.836 \mathrm{e}-6\end{array}$ & $\begin{array}{l}\text { NSGA-II } \\
3.602 \mathrm{e}-5\end{array}$ & $\begin{array}{l}\text { H-MOABC } \\
1.415 \mathrm{e}-1\end{array}$ & $\begin{array}{l}\text { CMOABC } \\
1.898 \mathrm{e}-1\end{array}$ & $\begin{array}{l}\text { NSGA-II } \\
1.992 \mathrm{e}-1\end{array}$ & $\begin{array}{l}\text { H-MOABC } \\
1.093 \mathrm{e}-2\end{array}$ & $\begin{array}{l}\text { CMOABC } \\
2.498 \mathrm{e}-2\end{array}$ & $\begin{array}{l}\text { NSGA-II } \\
3.098 \mathrm{e}-2 \\
\end{array}$ & $\begin{array}{l}\text { H-MOABC } \\
2.011 \mathrm{e}-2\end{array}$ & $\begin{array}{l}\text { CMOABC } \\
7.912 \mathrm{e}-2\end{array}$ & $\begin{array}{l}\text { NSGA-II } \\
9.376 \mathrm{e}-2\end{array}$ \\
\hline
\end{tabular}

Table 11. The best compromise solutions for each combination from the Pareto front based on involved algorithms on Rd500 (where $F_{l}, F_{2}$ and $F_{3}$ correspond to the first, second and third function in the combination respectively)

\begin{tabular}{|c|c|c|c|c|c|c|c|c|c|c|c|c|}
\hline & \multicolumn{3}{|c|}{$f_{1^{-}} f_{2^{-}} f_{3}$} & \multicolumn{3}{|c|}{$f_{1^{-}} f_{2^{-}} f_{4}$} & \multicolumn{3}{|c|}{$f_{1^{-}} f_{3^{-}} f_{4}$} & \multicolumn{3}{|c|}{$f_{2}-f_{3}-f_{4}$} \\
\hline & $\begin{array}{c}\mathrm{H}- \\
\text { MOABC }\end{array}$ & CMOABC & NSGA-II & $\begin{array}{c}\mathrm{H}- \\
\text { MOABC }\end{array}$ & CMOABC & $\begin{array}{c}\text { NSGA- } \\
\text { II }\end{array}$ & $\begin{array}{c}\mathrm{H}- \\
\text { MOABC }\end{array}$ & CMOABC & $\begin{array}{c}\text { NSGA- } \\
\text { II }\end{array}$ & $\begin{array}{c}\mathrm{H}- \\
\text { MOABC }\end{array}$ & CMOABC & NSGA-II \\
\hline$f_{1}$ & $4.793 e-6$ & $8.339 \mathrm{e}-6$ & $7.501 \mathrm{e}-5$ & $\begin{array}{l}3.512 \mathrm{e}- \\
5\end{array}$ & 5.482 e-5 & $\begin{array}{l}9.182 \mathrm{e}- \\
5\end{array}$ & $4.774 \mathrm{e}-5$ & $2.482 \mathrm{e}-5$ & $2.189 \mathrm{e}-5$ & $3.182 \mathrm{e}-1$ & 4.289 e-1 & $3.401 \mathrm{e}-1$ \\
\hline$f_{2}$ & 3.358 e- 1 & $4.024 \mathrm{e}-1$ & $7.362 \mathrm{e}-1$ & $\begin{array}{l}\text { 4. } 125 \mathrm{e}- \\
1\end{array}$ & $5.642 \mathrm{e}-1$ & $\begin{array}{l}6.711 \mathrm{e}- \\
1\end{array}$ & $1.498 \mathrm{e}-2$ & $1.982 \mathrm{e}-2$ & $\begin{array}{l}5.199 \mathrm{e}- \\
2\end{array}$ & $\begin{array}{l}9.011 \text { e- } \\
2\end{array}$ & 3. $552 \mathrm{e}-2$ & $1.152 \mathrm{e}-1$ \\
\hline$f_{3}$ & $1.702 \mathrm{e}-2$ & $3.189 \mathrm{e}-2$ & $4.834 \mathrm{e}-2$ & $4.387 \mathrm{e}-2$ & $5.559 \mathrm{e}-2$ & $\begin{array}{l}5.298 \text { e- } \\
2\end{array}$ & $5.872-2$ & $5.732 \mathrm{e}-2$ & $\begin{array}{l}3.201 \mathrm{e}- \\
2\end{array}$ & $5.401 \mathrm{e}-2$ & $5.901 \mathrm{e}-2$ & $3.199 \mathrm{e}-2$ \\
\hline \multirow[t]{3}{*}{$\begin{array}{l}\text { Reader } \\
\text { number }\end{array}$} & 39 & 45 & 46 & 43 & 45 & 45 & 40 & 40 & 39 & 42 & 45 & 47 \\
\hline & \multicolumn{3}{|c|}{$f_{1^{-}} f_{2}$} & \multicolumn{3}{|c|}{$f_{1}-f_{3}$} & \multicolumn{3}{|c|}{$f_{1}-f_{4}$} & \multicolumn{3}{|c|}{$f_{2}-f_{3}$} \\
\hline & $\begin{array}{c}\mathrm{H}- \\
\text { MOABC }\end{array}$ & CMOABC & NSGA-II & $\begin{array}{c}\mathrm{H}- \\
\text { MOABC }\end{array}$ & CMOABC & $\begin{array}{c}\text { NSGA- } \\
\text { II }\end{array}$ & $\begin{array}{c}\mathrm{H}- \\
\text { MOABC }\end{array}$ & CMOABC & $\begin{array}{c}\text { NSGA- } \\
\text { II }\end{array}$ & $\begin{array}{c}\mathrm{H}- \\
\text { MOABC }\end{array}$ & CMOABC & NSGA-II \\
\hline$f_{1}$ & $3.601 \mathrm{e}-6$ & $5.412 \mathrm{e}-5$ & 2.398 e-5 & $8.172 \mathrm{e}-5$ & $9.199 \mathrm{e}-6$ & $\begin{array}{l}9.230 \text { e- } \\
5\end{array}$ & $4.214 \mathrm{e}-6$ & $6.315 e-5$ & $\begin{array}{l}6.251 \mathrm{e}- \\
5\end{array}$ & $\begin{array}{l}1.121 \mathrm{e}- \\
1\end{array}$ & $2.298 \mathrm{e}-1$ & $5.001 \mathrm{e}-1$ \\
\hline$f_{2}$ & $2.301 \mathrm{e}-1$ & $2.934 \mathrm{e}-1$ & $5.183 \mathrm{e}-1$ & $\begin{array}{l}1.602 \mathrm{e}- \\
2\end{array}$ & $4.424 \mathrm{e}-2$ & $\begin{array}{l}6.712 \mathrm{e}- \\
2\end{array}$ & $5.682 \mathrm{e}-2$ & $6.84 \mathrm{e}-2$ & $\begin{array}{l}1.154 \mathrm{e}- \\
1\end{array}$ & $\begin{array}{l}2.198 \text { e- } \\
2\end{array}$ & $4.001 \mathrm{e}-2$ & $3.834 \mathrm{e}-2$ \\
\hline \multirow[t]{3}{*}{$\begin{array}{l}\text { Reader } \\
\text { number }\end{array}$} & 38 & 41 & 41 & 41 & 42 & 43 & 41 & 45 & 47 & 42 & 47 & 48 \\
\hline & & $f_{2}-f_{4}$ & & & $f_{3}-f_{4}$ & & & & & & & \\
\hline & $\begin{array}{c}\mathrm{H}- \\
\text { MOABC }\end{array}$ & CMOABC & NSGA-II & $\begin{array}{c}\mathrm{H}- \\
\text { MOABC }\end{array}$ & CMOABC & $\begin{array}{c}\text { NSGA- } \\
\text { II }\end{array}$ & & & & & & \\
\hline$f_{1}$ & $2.901 \mathrm{e}-1$ & $4.211 \mathrm{e}-1$ & $3.114 \mathrm{e}-1$ & $\begin{array}{l}1.146 \mathrm{e}- \\
2\end{array}$ & $1.253 \mathrm{e}-2$ & $\begin{array}{l}3.083 \text { e- } \\
2\end{array}$ & & & & & & \\
\hline$f_{2}$ & $6.204 \mathrm{e}-2$ & $6.143 \mathrm{e}-2$ & $6.934 \mathrm{e}-2$ & $5.702 \mathrm{e}-2$ & $6.224 \mathrm{e}-2$ & $\begin{array}{l}7.134 \text { e- } \\
2\end{array}$ & & & & & & \\
\hline $\begin{array}{l}\text { Reader } \\
\text { number }\end{array}$ & 39 & 42 & 45 & 39 & 41 & 41 & & & & & & \\
\hline
\end{tabular}




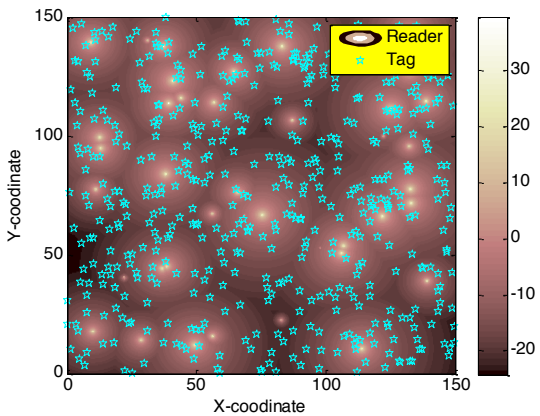

(a)

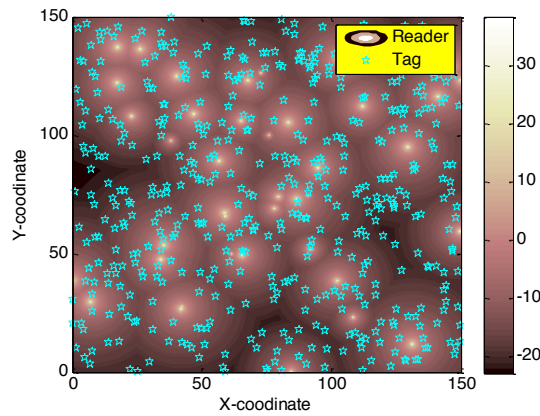

(b)

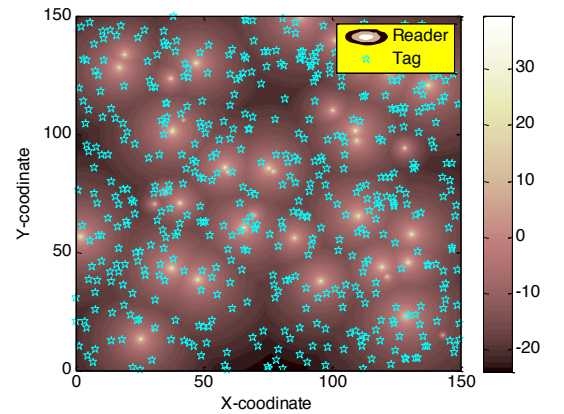

(c)

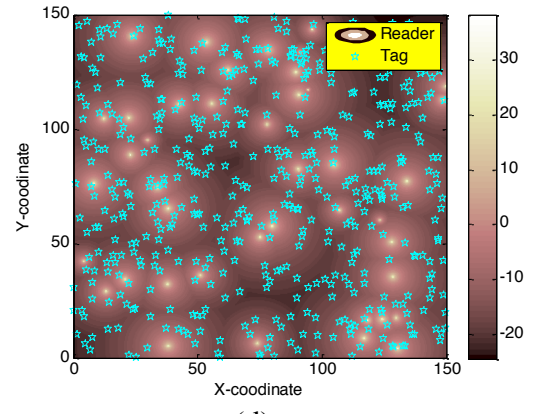

(d)

Fig.13. Reader distribution and radiated power contour obtained by H-MOABC: (a) for $f_{1}-f_{2}-f_{3}$, (b) for $f_{1}-f_{2}-f_{4}$, (c) for $f_{1}-f_{3}-f_{4}$, (d) for $f_{2}-f_{3}-f_{4}$

\subsubsection{Results on Rd500}

With the increasing number of readers and tags located in the working region increases, the optimization complexity of RNP increases exponentially. Accordingly, the Rd500 instance is employed to further investigate the algorithm's efficiency when handling large-scale scenarios. As shown in Fig. 9 (b), this can be formulated as a 150-dimensional bottom-level optimization problem. Similar to the previous section, all single-objective functions are firstly handled individually by $\mathrm{H}-\mathrm{MOABC}, \mathrm{CMOABC}$, and NSGA-II, as shown in Table 10. Then, Table 11 shows the bi-objective and tri-objective Pareto-optimal solutions obtained by H-MOABC, CMOABC and NSGA-II, where the associated bi-objective pairs include $f_{1}-f_{2}, f_{1}-f_{3}, f_{1}-f_{4}, f_{2}-f_{3}, f_{2}-f_{4}$, and $f_{3}-f_{4}$, and the associated tri-objective pairs include $f_{1}-f_{2}-f_{3}, f_{1}-f_{2}-f_{4}, f_{1}-f_{3}-f_{4}$, and $f_{2}-f_{3}-f_{4}$. Fig. 12 shows the reader locations and radiated power contours for the four three-objective instances in a visible way.

It can be observed from Table 11 that the objective function values obtained $\mathrm{H}-\mathrm{MOABC}$ are superior to those obtained CMOABC and NSGA-II on all biobjective and tri-objective functions instances, where obtained best compromise solutions are close to their optimal values as shown in Table 10. Furthermore, H-MOABC can provide an optimal reader layout via weeding out more redundant readers. As expected, by incorporating the effective dynamical crowded distance mechanism of H-MOABC, the high-density non-dominated solutions on large-scale RNP problem can be appropriately selected to be evolved in next growth iteration, leading to better results. The reader locations and radiated power contours obtained by $\mathrm{H}-\mathrm{MOABC}$ for four three-objective instances are plotted in Fig.13 It can be clearly observed from Fig.13 that HMOABC can provide a reasonable deployment scheme in the large-scale Rd500 instance.

\subsubsection{Discussion}

From above experimental results, it is apparent that the two-level RNP optimization based on $\mathrm{MO}$ algorithms aims to offer an optimal reader layout scheme with redundant reader elimination, and it exhibits significant superiorities to previous works using fixed number of readers in the optimization as below:

First, the optimal deployed reader number in the RFID system is difficult to pre-estimate, whereas excessive amount of readers to be deployed is not costefficient, and on the other hand inadequate readers cannot guarantee the full tag coverage. In contrast, the two-level MORNP model allows the algorithm to dynamically adjust the reader number by adaptively eliminating the redundant ones during the optimization process, and then achieve full tag coverage by using an appropriate reader number. This is the reason why the proposed scheme outperforms other schemes with fixed reader number.

Second, the novel two-level RNP model decouples the RNP optimization into binary top-level scheduling and multi-objective continuous bottom-level configuration. This essentially simplifies network management and accordingly reduces the optimization complexity. From the optimization point of view, this model is genetic and extendible that a broad variety of multi-objective optimization algorithms can be adopted on this model.

Finally, different from the single-objective optimization, in this MORNP optimization two or three objectives are optimized simultaneously and then the corresponding trade-off is taken into account: (1) convergence to the Paretooptimal set and (2) the diversity over Pareto-optimal solution. The approach of performance indicator based fitness assignment and dynamical crowded distance method incorporated by the proposed algorithm can cater to these purposes of complex RNP optimization.

Although there are many RNP models handled by some other optimization algorithms, most of them are not based on multi-objective approach, but always transform multiple RNP objectives into a single-objective problem by weighted coefficient approach. Essentially, their processing principle is different from our proposed approach, which also indicates that our multi-objective $\mathrm{ABC}$ algorithm is more suitable to find a set of optimal trade-off solutions for multiple conflicting objectives simultaneously in one run. Second, we have compared the existing multi-objective algorithms that have been applied in RNP with our proposed H-MOABC. Experimental results show the effectiveness of $\mathrm{H}-\mathrm{MOABC}$

Certainly, we cannot assert that the proposed RNP model and H-MOABC are always suitable to the real application scenarios because some simplified RNP problems do not involve the mixed discrete and continuous control variables, or some RNP objectives different from our proposed ones, even cannot be grouped into two layers. To some extent, this increases the difficulty of the proposed model and algorithm for real applications. In our future work we will focus on real applications of the proposed scheme.

\section{ConClusions}

This paper develops multi-level RNP framework and two-level MORNP model to decouple the complex RNP optimization, in order to reduce the RNP optimization complexity. Then a novel indicator-based multi-objective optimizer (H-MOABC) derived from the foraging rules of artificial bee colony is developed to solve this MORNP. In H-MOABC, based on the principle of $\mathrm{ABC}$ paradigm, a new fitness assignment based on quality indicator is developed to drive evolution of population and external archive, and the orthogonal Latin squares and comprehensive learning aim to boost population diversity to avoid being trapped into local optima. When all solutions are processed, the dynamical crowded distance estimation is employed to drive evolution of population for the next iteration.

Then, the H-MOABC is compared against NSGA-II, MOEA/D, and SPEA2 with a suit of typical bio-objective and tri-objective test problems by conducting experiments. Finally, the two-level MORNP model is resolved by this algorithm with two typical instances. Computational results show the effectiveness and efficiency of the two-level RNP scheme based on H-MOABC. In future, we will focus on the following research issues on RNP: (1) extending the proposed RNP model to other IoT applications, such as WSN optimization; (2) considering the dynamic RNP model for the RFID system with dynamic tags and mobile readers; (3) developing dynamic MOABC algorithm to solve the dynamic RNP problems; (4) conducting comprehensive sensitivity analysis of parameters of the algorithm and investigating the algorithm complexity.

\section{Acknowledgements}

The authors would like to thank the editors and anonymous reviewers for their helpful comments and suggestions on improving the quality of this paper. This work is supported by National Natural Science Foundation of China under National Science Foundation for Distinguished Young Scholars of China under Grant 71325002; Grant No. 61503373, 61572123; Natural Science Foundation of Liaoning Province under Grand 2015020002; and Fundamental Research Funds for the Central Universities (N161705001)

\section{References}

[1] B. Carbunar, M.K. Ramanathan, M. Koyut, etc. Efficient tag detection in RFID systems. Journal of Parallel and Distributed Computing, 2009, vol.69, pp.180-196.

[2] T. H Chang,S. C. Hsu, T. C. Wang, A proposed model for measuring the aggregative risk degree of implementing an RFID digital campus system with the consistent fuzzy preference relations, Applied Mathematical Modelling,vol.37(5), pp. 2605-2622, 2013

[3] J. Tan, F. Kong, W. Liang, A 3D object model for wireless camera networks with network constraints, Transactions of the Institute of Measurement and Control, vol. 35(7): pp. 866-874, 2013.

[4] R. Y. Zhong, G. Q. Huang, S. Lan, Q. Y. Dai, T. Zhang, C. Xu, A two-level advanced production planning and scheduling model for RFID-enabled ubiquitous manufacturing, Advanced Engineering Informatics, 2015, 29(4), pp.799-812.

[5] S.Y. Lin, H. F. Tsai, Micro genetic algorithm with spatial crossover and correction schemes for constrained three-dimensional reader network planning, Expert Systems With Applications, 2016, pp.344-353.

[6] L. Tang, H. Cao, L. Zheng, N. Huang, RFID network planning for wireless manufacturing considering the detection uncertainty, Ifac Papersonline, 2015, vol. no.3, pp.406-411.

[7] H. Feng, J. Qi, Radio frequency identification networks planning using new hybrid evolutionary algorithm. ICACT Transaction Advanced Communication Technology, 2013, 2(1), 179-188

[8] Q. Guan, Y. Liu, Y.-P. Yang, and W.-S. Yu, Genetic approach for network planning in the RFID systems, in Proc. 6th Int. Conf. Intell.Syst. Des. Appl. (ISDA'06), 2006, vol. 2, pp. 567-572.

[9] H.N. Chen, Y.L. Zhu, K.Y. Hu. Multi-colony Bacteria Foraging Optimization with cell-to-cell communication for RFID Network Planning. Applied Soft Computing, 2010, vol.10(2), pp.539-547.

[10] S. Lu, S. Yu, A fuzzy k -coverage approach for RFID network planning using plant 
growth simulation algorithm, Journal of Network \& Computer Applications, 2014, 39(1), pp.280-291.

[11] I. Bhattacharya and U. K. Roy, Optimal placement of readers in an RFID network using particle swarm optimization, Int. J. Comput. Netw. Commun., vol. 2, no. 6, pp. 225-234, 2010.

12] V. K. Vishwa, F. T. S. Chan, N. Mishra, V. Kumar, Environmental integrated closed loop logistics model: An artificial bee colony approach[C]// International Conference on Supply Chain Management and Information Systems. IEEE, 2010:1-7.

[13] Vishwa V. Kumar, Felix T.S. Chan, A superiority search and optimisation algorithm to solve RFID and an environmental factor embedded closed loop logistics model, International Journal of Production Research, 2011, 49(16):48074831.

[14] V. V. Kumar, F. W. Liou, S. N. Balakrishnan, V. Kumar, Economical impact of RFID implementation in remanufacturing: a Chaos-based Interactive Artificial Bee Colony approach, Journal of Intelligent Manufacturing, 2015, 26(4):815-830

[15] Y J. Gong, M. Shen, J. Zhang, O. Kaynak, W. N. Chen, Z. H. Zhan, Optimizing RFID Network Planning by Using a Particle Swarm Optimization Algorithm With Redundant Reader Elimination, IEEE Trans. Ind. Inf., vol. 8, no. 4, pp. 900-912, Nov. 2012.

[16] L. Ma, K. Hu, Y. Zhu, H. Chen, Cooperative artificial bee colony algorithm for multi-objective RFID network planning, Journal of Network \& Computer Applications, 2014, vol.42,pp.143-162.

[17] W. Pan, S. Li, Q. Xie, et al., Multi-objective Optimization of RFID Network Based on Genetic Programming, Information Technology Journal, 2011, 10(12):24272433.

[18] J. H. Seok, J. Y. Lee, C. Oh, J. J. Lee, et al, RFID sensor deployment using differential evolution for indoor mobile robot localization, in Proc. IEEE/RSJ Int. Conf. Intell. Robots Syst., 2010, pp. 3719-3724.

[19] I. Bhattacharya and U. K. Roy, Optimal placement of readers in an RFID network using particle swarm optimization, Int. J. Comput. Netw. Commun., vol. 2, no. 6 , pp. 225-234, 2010

[20] H.F. Tsai, S. Y. Lin, Genetic algorithm for reader network planning, International Journal Advanced Computer Network Security, 2013, 3(2),216-219.

[21] H.N. Chen, Y.L. Zhu, K.Y. Hu. Multi-colony Bacteria Foraging Optimization with cell-to-cell communication for RFID Network Planning. Applied Soft Computing, 2010, vol.10(2), pp.539-547.

[22] Z. Li, J. Li, C. He, Artificial immune network-based anti-collision algorithm for dense RFID readers, Expert Systems with Applications, vol. 41(10):pp. 4798-4810, 2014

[23] Bacanin, N., Tuba, M., \&Strumberger, I.(2015). RFID network planning by ABC algorithm hybridized with heuristic for initial number and locations of readers. In Proceeding of the 17th UK SIM-AMSS international conference on modeling and simulation, 2015, pp.39-44

[24] G. Anandaligam, T.I. Friesz, 1992. Hierarchical optimization Annals of Operations Research 34

[25] Akay B.. Synchronous and asynchronous Pareto-based multi-objective Artificial Bee Colony algorithms. J Glob Optim, 2013, 57:415-445

[26] Omkar S.N., Senthilnath J., Khandelwal R., Narayana Naik G., Gopalakrishnan S. Artificial Bee Colony (ABC) for multi-objective design optimization of composite structures. 2011, Applied Soft Computing, 11: 489-499.

[27] Yi X, Zhou Y. A dynamic multi-colony artificial bee colony algorithm for multiobjective optimization, Applied Soft Computing, 2015, 35(1):766-785.

[28] Xiang Y, Zhou Y, Liu H. An elitism based multi-objective artificial bee colony algorithm. European Journal of Operational Research, 2015, 245(1):168-193.

[29] Huo Y, Zhuang Y, Gu J, et al. Elite-guided multi-objective artificial bee colony algorithm. Applied Soft Computing, 2015, 32:199-210.

[30] K. V. S. Rao, P. V. Nikitin, and S. F. Lam, "Antenna design for UHF RFID tags: a review and a practical application," IEEE Trans. Antennas Propag., vol. 53, no. 12 , pp. 3870-3876, Dec. 2005.

[31] Homberger J., Gehring H. A parallel two-phase metaheuristic for routing problems with time windows. Asia-Pacific JOper Res 13(1):35-47, 2001.

[32] Solomon M. Algorithms for the vehicle routing and scheduling problems with time window constraints. Oper Res 35(2):254-265, 1987.

[33] Karaboga, D., An idea based on honey bee swarm for numerical optimization. 2005, Technical report-tr06, Erciyes university, engineering faculty, computer engineering department

[34] C. Watkins, P. Dayan. Technical Note: Q-learning. Machine Learning, 1992, 8(3): 279 292.

[35] Math. Stat. Res. Group (1975) 'Chinese Acad. Sci., Orthogonal Design (in Chinese)', Beijing, China: People Education Pub.

[36] Zhu G, Kwong S. Gbest-guided artificial bee colony algorithm for numerical function optimization, Applied Mathematics \& Computation, 2010, 217(7):31663173.

[37] Zitzler E, Künzli S. Indicator-Based Selection in Multiobjective Search. Lecture Notes in Computer Science, 2004, 3242:832-842.

[38] K. Deb, A. Pratap, S. Agarwal, and T. Meyarivan, A fast and elitist multiobjective genetic algorithm: NSGA-II, IEEE Trans. Evol. Comput., vol. 6, no. 2, pp. 182 197, Apr. 2002.

[39] G. Peng, Y. W. Fang, W. S. Peng, C. Dong, X. Yang, Multi-objective particle optimization algorithm based on sharing-learning and dynamic crowding distance, Optik - International Journal for Light and Electron Optics, 2016.

[40] N. Chen, W. N. Chen, Y. J. Gong, et al. An Evolutionary Algorithm with DoubleLevel Archives for Multiobjective Optimization, IEEE Transactions on Cybernetics, 2015, 45(9):1851-1863.

[41] I. C. Parmee, D. Cvetkovic, Preferences and their application in evolutionary multiobjective optimization, IEEE Trans. Evol. Comput., 2002, 6(1): 42-57.

[42] M. S. Kiran, The continuous artificial bee colony algorithm for binary optimization, Applied Soft Computing, 2015, 33(C):15-23.

[43] M. S. evkli, A.R. Güner, A continuous particle swarm optimization algorithmfor uncapacitated facility location problems, Lect. Notes Comput. Sci. 4150(316-323) (2006) 316 .

[44] E. Zitzler, Evolutionary algorithms for multiobjective optimisation: methods and applications, PhD thesis, Swiss Federal Institute of Technology (ETH), Zurich, Switzerland, 1999.

45] Q. Zhang, W. Liu, H. Li, The performance of a new version of MOEA/D on CEC09 unconstrained MOP test instances. Evolutionary Computation, 2009. CEC '09.IEEE Congress on. IEEE, 2009:203-208.

[46] Deb K, Thiele L, Laumanns M, et al. Scalable test problems for evolutionary multiobjective optimization. Springer London, 2005.

[47] Zhang, Q. and H. Li, MOEA/D: A multiobjective evolutionary algorithm based on decomposition. Evolutionary Computation, IEEE Transactions on, 2007. 11(6): p. 712-731.

[48] Zitzler E, Laumanns M, Thiele L, et al. SPEA2: Improving the strength Pareto evolutionary algorithm. Evolutionary Methods for Design Optimization and Control with Applications to Industrial Problems, 2001:95-100.

[49] Akay B. "Synchronous and asynchronous Pareto-based multi-objective Artificial Bee Colony algorithms," Journal of Global Optimization, 2013, 57(2): 415-445

[50] Wenping Zou, Yunlong Zhu, Hanning Chen, and Beiwei Zhang,"Solving MultiObjective Optimization Problems Using Artificial Bee Colony Algorithm", Discrete Dynamics in Nature and Society, 2011, 2011(2):1-37.

[51] W. J. Connover, Practical Nonparametric Statistics, 3rd ed. New York, NY, USA: Wiley, 1999, ch. 5.

52] Lin S Y, Tsai H F. Micro genetic algorithm with spatial crossover and correction schemes for constrained three-dimensional reader network planning, Expert Systems with Applications, 2016, 44(C):344-353

[53] C. L. Philip Chen, T. Zhang, L. Chen, and S. K. Tam, I-Ching Divination Evolutionary Algorithm and its Convergence Analysis, IEEE Transactions On Cybernetics, 47(1), pp. 2-13, 2017.

[54] M. Gong, L. Jiao, H. Du, L. Bo, Multiobjective immune algorithm with nondominated neighbor-based selection, Evolutionary Computation, 2008, 16(2):225 - 255.

[55] F. Santoso. A New Framework for Rapid Wireless Tracking Verifications Based on Optimized Trajectories in Received Signal Strength Measurements, IEEE Transactions on Systems Man \& Cybernetics Systems, 2015, 45(11):1-1.

[56] D. Li, C. Zhang, G. Tian, X. Shao, Z. Li. Multiobjective Program and Hybrid Imperialist Competitive Algorithm for the Mixed-Model Two-Sided Assembly Lines Subject to Multiple Constraints, IEEE Transactions on Systems Man \& Cybernetics Systems, 2016, PP(99):1-11.

[57] Zheng X L, Wang L. A Collaborative Multiobjective Fruit Fly Optimization Algorithm for the Resource Constrained Unrelated Parallel Machine Green Scheduling Problem, IEEE Transactions on Systems Man \& Cybernetics Systems, 2016, PP(99):1-11.

[58] Y. Hou, N. Q. Wu, M. C. Zhou, Z.W. Li. Pareto-Optimization for Scheduling of Crude Oil Operations in Refinery via Genetic Algorithm, IEEE Transactions on Systems Man \& Cybernetics Systems, 2015, 12(2-3):1-14

[59] A. N. K. Nasir, M. O. Tokhi. An Improved Spiral Dynamic Optimization Algorithm With Engineering Application, IEEE Transactions on Systems Man \& Cybernetics Systems, 2015, 45(6):1-1

[60] E. Zitzler, L. Thiele, M. Laumanns, et al. Performance assessment of multiobjective optimizers: an analysis and review. IEEE Transactions on Evolutionary Computation, 2003, 7(2):117-132.

[61] R. Cheng, Y. Jin, M. Olhofer, et al. A Reference Vector Guided Evolutionary Algorithm for Many-Objective Optimization. IEEE Transactions on Evolutionary Computation, 2016, 20.

[62] C. L. Philip Chen, T. Zhang, L. Chen, and S. K. Tam, I-Ching Divination Evolutionary Algorithm and its Convergence Analysis, IEEE Transactions On Cybernetics, 2017, 47(1): 2-13.

[63] C. L. Philip Chen, J. Zhou, and W. Zhao, A Real-Time Vehicle Navigation Algorithm in Sensor Network Environments, IEEE Trans. On Intelligent Transportation Systems, 2012, 13(4):1657-1666 